\title{
ILCEA
}

Revue de l'Institut des langues et cultures

d'Europe, Amérique, Afrique, Asie et Australie

9| 2007

L'implicite et les écrits de l'entreprise

\section{L'implicite dans le discours des grandes entreprises sur leur responsabilité sociale}

\section{Catherine Resche}

\section{OpenEdition \\ Journals}

Édition électronique

URL : http://journals.openedition.org/ilcea/626

DOI : 10.4000/ilcea.626

ISSN : 2101-0609

\section{Éditeur}

UGA Éditions/Université Grenoble Alpes

\section{Édition imprimée}

Date de publication : 15 décembre 2007

Pagination : 7-47

ISBN : 978-2-84310-104-5

ISSN : 1639-6073

Référence électronique

Catherine Resche, "L'implicite dans le discours des grandes entreprises sur leur responsabilité sociale », ILCEA [En ligne], 9 | 2007, mis en ligne le 09 juin 2010, consulté le 01 mai 2019. URL : http:// journals.openedition.org/ilcea/626; DOI : 10.4000/ilcea.626 


\title{
L'implicite dans le discours des grandes entreprises sur leur responsabilité sociale
}

\author{
Catherine REsCHE \\ Université Panthéon-Assas - Paris 2
}

\begin{abstract}
RÉSUMÉ
Cette étude s'intéresse au message implicite contenu dans le discours des grandes entreprises sur leur responsabilité sociale. Les entreprises dont il s'agit ont été sélectionnées pour entrer dans la composition de deux indices éthiques connus le FTSE4Good et le DJSI. Après l'analyse des termes phares des sites en ligne de ces entreprises, l'implicite est étudié au niveau des dénominations des notions dans un premier temps, puis au niveau du discours des dirigeants dans leurs introductions aux rapports annuels sur la responsabilité des entreprises. Si l'implicite se situe au niveau des présupposés et des connotations au niveau des termes, c'est sous la forme d'implicatures qu'il peut être décelé dans le discours. Le discours des entreprises s'avère être un discours de légitimation de l'économie de marché.
\end{abstract}

\section{ABSTRACT}

This paper focuses on the implicit message in corporate responsibility discourse by the firms selected by two well-known ethical indices - FTSE4Good and DFSI. The key terms emerging from the companies' websites are first identified and analysed, then the implicit message contained in the main notions is discussed; finally, the discourse of the companies' managers and CEOs is scrutinised in order to make clear its implicit message. While terms are characterized by connotations and presuppositions, implicit discourse is better understood through implicatures. Corporate discourse turns out to be aimed at legitimising our market economy.

Mots-clés - Implicite, discours, responsabilité sociale des entreprises, citoyenneté des entreprises, rapports.

Key words -Implicit message, discourse, Corporate Social Responsibility, Corporate Citizenship, reports. 
Cette étude s'inscrit dans le prolongement d'une première étude (Resche 2005) qui s'intéressait au genre des mission statements des grandes entreprises cotées en bourse. Il convient de rappeler que nous avions établi que cette appellation, aussi répandue soit-elle dans le monde des entreprises, ne correspond pas à une définition très stricte. Pour la cerner, il nous avait fallu rassembler un certain nombre de textes permettant aux entreprises d'annoncer leur mission, leur vision, leurs croyances, principes et valeurs. Si, dans notre étude préliminaire, nous nous étions attachée à déterminer ce qui influence le discours des grandes entreprises dans la déclaration de leur mission, nous souhaiterions maintenant orienter notre nouvelle analyse vers un aspect bien particulier en nous intéressant au discours sur la responsabilité sociale des entreprises. À cet égard, nous aimerions préciser que la métonymie « discours des grandes entreprises» contenue dans la première partie de notre titre est voulue, et nous aurons l'occasion de revenir sur ce point. L'idée selon laquelle la responsabilité des entreprises va bien au-delà de leur devoir de rentabilité envers leurs actionnaires n'est pas nouvelle (Hindle 2003 : 44). En effet, dès le XIX ${ }^{e}$ siècle, il n'était pas rare que les entreprises construisent des logements spécialement destinés à leurs ouvriers, au motif qu'un logement décent avait pour effet d'améliorer le rendement et la qualité du travail de la main-d'œuvre. N'oublions pas non plus que dans les années 1880 aux États-Unis, les «Robber Barons» n'étaient pas uniquement préoccupés par les bénéfices à réaliser. Andrew Carnegie, qui a fait fortune dans l'industrie de l'acier à Pittsburgh, a aussi associé son nom à la création de bibliothèques. Rockefeller, de son côté, fit de la Standard Oil Company un immense trust et consacra une partie de sa fortune à des activités philanthropiques. Un siècle plus tard, dans les années 1980, le débat avait évolué vers ce qu'on a appelé l'entreprise éthique et en 1987, Sir Adrian Cadbury écrivait dans le Harvard Business Review:

Les chefs d'entreprises ont toujours été confrontés à la difficulté de concilier les considérations éthiques et les préoccupations commerciales. Ce problème n'est pas nouveau. La différence aujourd'hui est que nos décisions et les jugements éthiques sous-jacents font l'objet d'un intérêt plus large et d'une analyse critique $^{1}$.

Comme le précise le Better Business Bureau aux États-Unis, non seulement, un manquement à l'éthique peut être préjudiciable à une entreprise, mais il peut être préjudiciable à l'ensemble des entreprises, au

1. The possibility that ethical and commercial considerations will conflict has always faced those who run companies. It is not a new problem. The difference now is that a more widespread and critical interest is being taken in our decisions and in the ethical judgments which lie behind them. 
monde des affaires. Avec la mondialisation des échanges, il est évident que les grandes entreprises internationales ne peuvent ignorer ce point et les nouveaux enjeux.

Notre propos, dans cette seconde étude, est de nous attacher non pas à ce qui est dit, mais plutôt à ce qui n'est pas dit, ce qui est implicite ou insinué, aux omissions éventuelles, allusions ou suggestions, à leur effet et au message qui est ainsi insidieusement transmis.

Pour mener à bien cette étude, nous évoquerons d'abord le cadre théorique qui a guidé notre approche, puis nous expliquerons le choix de notre corpus et notre méthodologie, avant de livrer nos réflexions et conclusions sur l'implicite au niveau des termes, donc des notions, puis du discours. Le cadre de cet article étant forcément restreint, nous avons décidé de nous attacher, pour l'analyse du discours, aux rapports spéciaux dans lesquels les entreprises rendent compte de leur bilan en matière de responsabilité sociale et environnementale, en nous limitant aux introductions rédigées par les responsables.

\section{Cadre théorique : l'implicite}

La définition de l'implicite telle qu'elle est donnée par le Robert (1977: 628) souligne que «Implicite» vient du latin implicitus, qui signifie étymologiquement «enveloppé» et renvoie à ce qui est sous-entendu. «Est implicite ce qui est virtuellement contenu dans une proposition, un fait, sans être formellement exprimé, et peut en être tiré par voie de conséquence, par déduction, induction, etc.».

La première question qu'il nous faut poser est la raison à ce sousentendu. Ce qui est tu, peut l'être intentionnellement pour ne pas blesser, pour se protéger, ou par respect des conventions. Il peut également s'agir d'un non-dit qui n'a pas besoin d'être dit, parce que la chose est évidente, connue, qu'il s'agit d'un implicite culturel. La logique peut aussi être mobilisée pour amener l'autre à accepter comme évident ce qui ne l'était pas au départ. Dans ce cas, on tente d'influencer l'autre, de le manipuler parfois. On peut également considérer l'implicite comme insinuation, connotation, filigrane, arrière pensée, évocation, inférence. Ce qui est inexprimé peut enfin être présupposé ou impliqué. Il nous faut ici établir une distinction entre présupposés et implicatures (Grice 1978): les présupposés sont des idées considérées comme acquises et sont inhérents aux termes utilisés par le locuteur/auteur, alors que les implicatures ne sont pas contenues dans les termes utilisés, mais simplement déduites de la façon dont les phrases sont construites et les idées amenées. On pourrait donc avancer que si les présupposés sont à envisager aussi bien au niveau des termes que du discours, c'est à ce dernier niveau qu'il nous faudra nous appliquer à trouver les implicatures. 
Si nous analysons le titre choisi pour cette étude, nous pouvons y trouver deux manifestions de l'implicite: le procédé métonymique qui consiste à réduire l'expression «dirigeants des entreprises» à «entreprises $\gg$ conduit à une personnification et présuppose que ces entreprises se confondent avec des personnes. Elles sont capables de tenir un discours, avec tout ce que cela peut signifier en termes d'influence et de rôle à jouer. Se pose déjà la question de l'entreprise comme acteur. La deuxième manifestation de l'implicite réside dans le fait de parler de responsabilité sociale des entreprises comme s'il était acquis que les entreprises ont un rôle social à jouer. Nous nous intéresserons à cette forme d'implicite dans l'analyse des termes clés de notre corpus.

Les risques existent de ne pas toujours saisir l'information implicite, l'allusion, de passer à côté d'une partie du message ou de se laisser influencer à son insu; il est également possible de mal décoder l'implicite, ce qui peut aboutir à des malentendus. Quoi qu'il en soit, quiconque cherche à analyser un discours ne peut ignorer les présupposés et sousentendus, autrement dit l'implicite, qui participent également à la construction du sens. La prise en compte du contexte immédiat du texte, de la situation d'énonciation, et du contexte plus large, doit conduire à une meilleure compréhension des énoncés. Comme le souligne KerbratOrecchioni (Charaudeau \& Maingueneau 2002 : 306), «les discours agissent aussi, subrepticement mais efficacement, grâce à ces sortes de passagers clandestins que sont les contenus implicites». Nous avancerons, en empruntant à Austin (1970) que si « dire, c'est faire», ne pas dire est aussi agir. Les silences ou absences ont donc un sens que nous nous sommes attachée à faire ressortir en analysant les textes réunis dans notre corpus.

\section{Corpus}

Nous avons souhaité constituer un corpus homogène et, pour ce faire, nous avons fondé notre étude sur deux indices particuliers, qui rassemblent des entreprises sélectionnées sur des critères bien définis. Ces entreprises considèrent que leur inclusion dans ces indices est une marque de reconnaissance, une sorte de label de qualité pour entreprises soucieuses de leurs responsabilités sociales et environnementales. Il s'agit du FTSE4Good UK 50 pour le Royaume Uni et du DFSI US 50 (Dow fones Sustainability Index) pour les États-Unis. L'avantage est que ces deux indices sont de taille égale (50 entreprises chacun) et qu'ils sont désormais bien connus des investisseurs et autres parties prenantes. Il va de soi que la liste des entreprises composant chaque indice est régulièrement soumise à révision et qu'aucune entreprise ne peut se permettre de 
se reposer sur ses lauriers une fois qu'elle a été couronnée; le défi est constant de conserver cette reconnaissance et d'élever sans cesse le niveau de responsabilité sociale. Il est intéressant de comparer les critères annoncés en ligne par chacun des indices ci-dessous:

\section{Caractéristiques}

- Des critères évolutifs qui reflètent toujours un large consensus du marché en matière de responsabilité sociale et code de bonne conduite des entreprises

- Des critères exigeants, mais réalistes qui encouragent les entreprises à faire des efforts pour les respecter

- Des normes plus strictes pour les entreprises dont l'impact est plus important

- Des critères et une méthodologie clairement exposés

- Des critères qui s'appuient sur des codes et des principes universellement reconnus; les nouveaux critères sont mis au point au terme d'un processus de consultation mondiale avant d'être approuvés par un comité consultatif indépendant

Pour être admises, les sociétés doivent satisfaire à cinq conditions dans les domaines suivants :

- Travailler à la protection durable de l'environnement

- Développer des relations positives avec l'ensemble des parties prenantes

- CEuvrer à la promotion et à la protection des droits de l'homme

- Établir et respecter des normes valables en ce qui concerne l'ensemble des fournisseurs et travailleurs

- Lutter contre la corruption ${ }^{2}$ (FTSE4Good)

Critères d'évaluation pour une entreprise durable: Ces critères ont été établis à l'issue d'une évaluation des éléments moteurs et des tendances au niveau de l'impact économique, environnemental et sociétal. Ces critères ont été arrêtés pour chacun de ces impacts et pour tous les secteurs d'activités. On distingue les critères généraux, qui s'appliquent à tous les secteurs, et les critères spécifiques à tel ou tel secteur. [...]

Les critères généraux comprennent les pratiques classiques de gestion et les mesures de l'activité valables pour tous les secteurs: la gouvernance d'entreprise, la gestion de la chaîne des fournisseurs au niveau environnemental, la gestion du risque, la gestion de crise, et les pratiques en matière de gestion du personnel. Ces critères généraux comptent pour moitié dans l'évaluation.

\section{Key features:}

Evolving selection criteria to reflect changes in globally accepted corporate responsibility standards and codes of conduct over time.

Challenging yet achievable criteria that encourage companies to strive to meet them.

Higher impact companies have to meet higher standards.

Transparent criteria and methodology.

Criteria based on internationally respected codes and principles with new criteria subjected to a widespread consultation and approved by an independent oversight committee.

[...]For inclusion, eligible companies must meet criteria requirements in five areas:

Working towards environmental sustainability

Developing positive relationships with stakeholders

Up-holding and supporting universal human rights

Ensuring good supply chain labour standards

Countering bribery (FTSE4Good) 
[...] Les critères spécifiques à tel ou tel secteur tiennent compte des défis et des tendances propres au secteur en question. Ils [...] comptent pour l'autre moitié dans l'évaluation $^{3}$. (DJSI)

La lecture de ces explications permet de mieux apprécier ce que signifient les noms des deux indices, ce qu'il faut inclure, par exemple, dans l'enveloppe «for Good» pour le FTSE. Bien que le terme Sustainability soit inclus dans le nom du DJSI, on constate que cet indice n'est pas exclusivement concerné par la notion de durabilité. En réalité, la comparaison des critères annoncés par les deux indices fait ressortir des préoccupations proches, qui se traduisent par les mêmes grandes catégories relevant de considérations économiques, environnementales et sociales. Dans les deux cas, les notions de gouvernance, de bonnes pratiques, de prise en compte des diverses parties prenantes sont mentionnées. Si les extraits choisis ci-dessus semblent indiquer une préoccupation plus explicite du FTSE pour le respect des droits de l'homme et la lutte contre la corruption, ces mêmes thèmes sont abordés ailleurs pour le DJSI, de sorte que, malgré leur appellation différente, les deux indices ont des critères proches. Il n'est d'ailleurs pas rare qu'une même entreprise annonce qu'elle a été reconnue par l'un et l'autre.

Une fois la composition de chaque indice connue (en date de février 2007), nous avons systématiquement consulté les sites en ligne des entreprises afin de réunir les textes à prendre en compte dans notre analyse. Cette consultation nous a permis de prendre conscience d'une première difficulté: chaque site est particulier, et, si certains proposent directement une rubrique «Corporate Social Responsibility» ou simplement «Corporate Responsibility», d'autres proposent des rubriques ne contenant pas ces termes, et forcent à explorer le site pour trouver les renseignements nécessaires. Cette première difficulté nous a conduite à nous poser la question de savoir si nous devions ne retenir que les rubriques qui contenaient les termes «responsibility» ou «sustainability», ou s'il convenait d'être plus accueillante dans un premier temps, pour éviter le risque de passer à côté d'éléments qui relevaient de notre sujet

3. Corporate Sustainability Assessment Criteria: Through the assessment of economic, environmental and social driving forces and trends, corporate sustainability criteria are identified. Criteria are identified for each dimension and for all industries. In addition, the criteria are defined as either general criteria applicable to all industries or industry specific criteria. [...] [General criteria] include standard management practices and performance measures applicable to all industries, such as corporate governance, environmental supply chain management, risk and crisis management and labor practices. The general criteria account for approximately fifty percent of the assessment. [...] Industry specific criteria take into account the challenges and trends affecting specific industries. They $[\ldots]$ have a weight of approximately fifty percent of the assessment. [...] (DJSI) 
d'étude, sans l'annoncer directement. Une première aide nous est venue des notions clés qui ressortent des critères des deux indices, et nous avons également souhaité confronter ces notions à celles qui sont portées par les définitions sur lesquelles nous reviendrons. Ceci nous a permis de procéder à des recoupements et de déterminer avec certitude quelles rubriques consulter et inclure dans notre corpus. Pour donner au lecteur une idée de ce réseau notionnel, nous avons établi un tableau (Annexe 1) des rubriques principales et sous-rubriques pour chaque entreprise. Il nous est apparu instructif de faire figurer les sousrubriques pour montrer les relations d'hypéronymie ou d'hyponymie entre les termes. L'observation des arborescences ainsi créées de manière implicite par les entreprises nous a permis de comparer les deux indices et la façon dont leurs composantes concevaient leurs responsabilités et organisaient leur façon de communiquer sur ce sujet. Avant de livrer et d'analyser les résultats de notre première recherche de l'implicite au niveau du choix des termes, il convient de revenir sur un certain nombre de définitions qui nous ont permis de délimiter notre corpus.

\section{Définitions}

Notre intention première, en nous fondant sur les définitions des termes les plus fréquemment rencontrés, était de chercher à comprendre comment s'organise la terminologie propre au domaine de la responsabilité sociale des entreprises. Dans la mesure où la définition d'un terme, unité minimale de sens dans les domaines de spécialité, doit permettre de délimiter sa surface notionnelle, notre propos était de parvenir à établir une carte notionnelle du domaine. Les définitions ci-dessous ont pour source des articles de recherche en gestion des entreprises, des sites spécialisés ou encore les entreprises elles-mêmes. Nous avons vite pris conscience que l'implicite était déjà sensible à ce niveau, puisque le flou qui entoure les définitions laisse la place à une certaine interprétation plus personnelle.

Commençons par la notion «Corporate Social Responsibility» ou «responsabilité sociale de l'entreprise» (RSE). Pour Cramer et al. (2004:1), cette notion s'articule selon trois axes à savoir, les hommes, l'environnement et la rentabilité; elle vient en écho aux trois domaines précisés dans les critères des deux indices:

La responsabilité sociale de l'entreprise (RSE) est en passe de devenir une question clé pour les entreprises. Elle intéresse des entreprises qui recherchent sciemment à créer de la valeur dans leurs activités dans trois directions: au niveau des hommes (en assurant le bien-être de ceux qui travaillent au sein de l'entreprise mais aussi d'une communauté plus large); au niveau de la planète (en 
cherchant à améliorer la qualité de l'environnement) et au niveau de la rentabilité (en cherchant à maximaliser les bénéfices). Ce faisant, ces entreprises soignent leurs relations avec les diverses parties prenantes en communiquant de manière claire et transparente et en maintenant le dialogue ${ }^{4}$.

À partir de la mention de la Planète et de la nécessité d'engranger des profits, donc de survivre, on peut déduire de cette définition la notion de durabilité, qui est clairement exprimée dans la définition de la responsabilité sociale des entreprises fournie par Marks \& Spencer:

Qu'entendons-nous par RSE? La responsabilité sociale de l'entreprise est souvent présentée en termes d'écoute des parties prenantes de l'entreprise et de réponse à leurs besoins. Ceci implique la prise en compte des exigences en matière de développement durable. Nous pensons que la meilleure garantie de la réussite à long terme est de soigner les relations que nous entretenons avec nos employés, nos fournisseurs et la société au sens large. C'est la ligne directrice de notre approche en matière de $\mathrm{RSE}^{5}$.

Peut-on vraiment trouver une différence aussi significative que celle que l'on pourrait attendre si l'on retire «social » de la dénomination, comme le fait BG group?

La responsabilité de l'entreprise (RE) est un terme répandu qui est ouvert à de nombreuses interprétations. Pour le groupe BG, la RE décrit l'essentiel de notre conception de l'entreprise. Notre objet est de nous hisser au plus haut niveau d'exigence en matière de bonne conduite, dans notre comportement envers nos gens et ceux qui traitent avec nous, dans nos relations avec les communautés, et dans notre respect exemplaire de l'environnement ${ }^{6}$. (Groupe BG)

Encore une fois, on retrouve les notions clés, exprimées différemment: l'adjectif social est en filigrane derrière les notions de relations, de bonne conduite, de la manière dont les gens et les choses sont traitées. De tout cela dépend la durabilité de l'entreprise, et de son environnement humain ou naturel.

4. Corporate social responsibility (CSR) is becoming a key issue for business. It involves companies consciously orienting their activities towards value creation in three dimensions - People (creation of well-being in and outside the organisation), Planet (achievement of ecological quality) and Profit (maximization of profit) -while at the same time maintaining a clear and communicative relationship with the diverse stakeholders on the basis of transparency and dialogue.

5. What CSR means to us: Corporate Social Responsibility is often described as listening and responding to the needs of a company's stakeholders. This includes the requirements of sustainable development. We believe that building good relationships with employees, suppliers and wider society is the best guarantee of long-term success. This is the backbone of our approach to CSR.

6. Corporate Responsibility (CR) is a widely used term which is open to many interpretations. In BG Group, CR describes our core approach to business. Our aim is adherence to the highest standards of business conduct, world-class treatment of our people and contractors, good relationships with communities and exemplary environmental performance. (BG group) 
Intéressons-nous maintenant à l'étiquette «corporate sustainability» pour établir les correspondances ou différences éventuelles avec les définitions précédentes. La définition qu'en donnent les consultants du groupe Article $13^{7}$ fait étrangement écho aux définitions des autres notions citées plus haut:

La durabilité de l'entreprise (Corporate sustainability): la capacité pour une organisation de poursuivre ses activités indéfiniment, en ayant pris en compte leur impact sur le capital financier, social et environnemental. Les organisations incorporent les critères économiques, environnementaux et sociaux à leur stratégie et à leur gestion. Elles cherchent à saisir les occasions qui se présentent et gèrent le risque. Elles créent de la valeur à long terme pour leurs actionnaires en s'engageant fermement à viser les meilleurs résultats tout en gardant à l'esprit toutes les autres considérations dans la conduite de leurs activités ${ }^{8}$.

Pour ce même groupe, une notion supplémentaire est présentée comme l'émanation du développement durable; il s'agit de la citoyenneté de l'entreprise «Corporate Citizenship», définie comme suit:

Une approche par laquelle une entreprise:

- reconnaît que ses activités ont un impact plus large sur la société au sein de laquelle elle est installée, et que les évolutions de la société ont également un impact sur sa capacité à poursuivre avec succès ses activités;

- gère efficacement l'impact économique, social, environnemental que ses activités ont de par le monde, y compris en ce qui concerne les droits de l'bomme, en s'appuyant sur les principes qui traduisent les valeurs universelles. Les bénéfices qu'elle tirera de tout ceci profiteront aussi bien à l'entreprise et à sa réputation, qu'aux communautés dans lesquelles elle exerce ses activités;

- cherche à récolter ces bénéfices en collaborant étroitement avec d'autres groupes et organisations - les communautés locales, la société civile, d'autres entreprises, ainsi que son gouvernement ou les gouvernements des pays qui l'accueillent.

Cette approche est inspirée des principes du développement durable ${ }^{9}$.

7. <www.article13.com>

8. Corporate sustainability: The capability of an organisation to continue its activities indefinitely, having taken account of their impact on financial, social and environmental capitals. Organisations integrate economic, environmental and social criteria into strategy and management. They pursue opportunities and manage risks. They create longterm shareholder value by leading their industry with a strong commitment and superior performance in all these dimensions.

9. An approach by which a company: recognises that its activities have a wider impact on the society in which it operates; and that developments in society in turn impact on its ability to pursue its business successfully; actively manages the economic, social, environmental and human rights impact of its activities across the world, basing these on principles which reflect international values, reaping benefits both for its own operations and reputation as well as for the communities in which it operates; seeks to achieve these benefits by working closely with other groups and organisations - local communities, civil society, other businesses and home and host governments. This approach is derived from principles of sustainable development. 
Du coté des entreprises, les notions sont reprises comme un leitmotiv. Nous citerons en illustration Abbott Laboratories, une des composantes du DJSI US 50:

La citoyenneté planétaire traduit la façon dont une entreprise poursuit ses objectifs, implique les parties prenantes, met en œuvre sa politique, pratique l'investissement social et la philanthropie, et joue de son influence pour apporter sa contribution efficace à la société. Chez Abbott, la citoyenneté planétaire correspond également à la recherche d'un équilibre raisonné entre les responsabilités financières, environnementales et sociales, et le souci de contribuer à la qualité de la santé et des soins partout dans le monde. Nos programmes comprennent l'instruction publique, l'environnement, la santé et la sécurité, et l'accès aux soins. Ces efforts traduisent notre implication et notre partenariat avec les parties prenantes pour rechercher des solutions durables aux défis que l'ensemble de la planète doit relever ${ }^{10}$.

McIntosh et al (1998 : 16) nous offrent une dernière définition qui semble insister davantage sur l'idée que les entreprises sont des membres de la société, point sur lequel nous reviendrons, mais reprend également les notions omniprésentes des responsabilités en matière d'impact social et environnemental

La citoyenneté de l'entreprise [...] concerne des entreprises qui sont de plus en plus informées, et des membres éclairés de la société civile. Ces entreprises comprennent qu'elles sont des entités à la fois publiques et privées. [...] Elles sont un produit de la société et elles tirent leur légitimité des sociétés dans lesquelles elles exercent leurs activités. Il est nécessaire qu'elles définissent clairement leur rôle, leur champ d'action et leur but et qu'elles prennent conscience de leur impact sur l'environnement et la société, et des responsabilités qui sont les leurs en la matière ${ }^{11}$.

Tous les termes qui figurent en italiques dans les citations ci-dessus sont de notre fait, pour attirer l'attention du lecteur sur les recoupements d'une définition à l'autre. De toute évidence, nous sommes en présence d'un faisceau de notions proches, comme l'ont souligné les chercheurs à maintes reprises (Carroll, 1998, 1999; Garriga \& Melé, 2004; Swanson,

10. Global citizenship reflects how a company advances its business objectives, engages its stakeholders, implements its policies, applies its social investment and philanthropy, and exercises its influence to make productive contributions to society. At Abbott, global citizenship also means thoughtfully balancing financial, environmental and social responsibilities with providing quality health care worldwide. Our programs include public education; environment, health and safety; and access to health care. These efforts reflect an engagement and partnership with stakeholders in the pursuit of sustainable solutions to challenges facing the global community.

11. Corporate Citizenship [...] involves corporations becoming more informed and enlightened members of society and understanding that they are both public and private entities. [...] They are created by society and derive their legitimacy from the societies in which they operate. They need to be able to articulate their role, scope and purpose as well as understand their full social and environmental impacts and responsibilities. 
1995). Nous verrons bientôt, en nous attachant à débusquer l'implicite derrière le choix des termes, qu'il y a des nuances, mais, pour conclure ce rapide tour d'horizon, nous citerons une dernière définition qui nous semble bien résumer la difficulté à établir des délimitations claires des surfaces notionnelles de ces termes. Elle revient sur la RSE et émane du cabinet Juno ${ }^{12}$, consultants en gestion des entreprises, spécialisés dans la RSE:

En dépit de l'importance croissante de la RSE, il n'y a pas de consensus quant au sens exact de l'expression et il existe de nombreuses appellations pour des concepts équivalents ou proches, comme par exemple, la citoyenneté de l'entreprise, la durabilité de l'entreprise et la responsabilité de l'entreprise.

Bien que certains débattent des nuances entre ces termes, sur le fond, tout le monde s'accorde sur le même principe fondamental: la responsabilité d'une entreprise va au-delà des bénéfices qu'elle réalise pour ses actionnaires. Elle se doit, entre autres responsabilités, de bien traiter ses employés, de respecter l'environnement, de mettre en place un système solide de gouvernance d'entreprise, d'encourager la philanthropie, de renforcer les droits de l'homme, de respecter les différences culturelles et d'aider à promouvoir le commerce équitable. Toutes ces actions sont censées avoir un impact bénéfique sur les communautés, cultures, sociétés et lieux dans lesquels les entreprises exercent leurs activités.

Ces efforts devraient également bénéficier aux différentes parties prenantes, qui comprennent tout ou partie des groupes suivants: clients, employés, cadres de direction, membres extérieurs des conseils de direction, investisseurs, créditeurs, vendeurs, fournisseurs, gouvernements, ONG, communautés locales, défenseurs de l'environnement, organisations caritatives, autochtones, fondations, groupes religieux, et organisations culturelles ${ }^{13}$.

Forte de ce constat de quasi-similitude entre les diverses dénominations, nous avons donc décidé d'inclure dans notre corpus toutes les rubriques correspondant aux termes clés contenus dans les définitions. À partir de là, en analysant les arborescences réalisées grâce à l'observation des

\section{2. <www.junoconsulting.com>}

13. Despite the growing importance of CSR, there is little agreement as to what the phrase really means and there are many different names for the same or similar concept, such as Corporate Citizenship, Business Sustainability and Corporate Responsibility.

While some may argue over the distinctions among these terms, at the core they all point towards the same fundamental principle: that a company is responsible for providing more benefits than just profits for shareholders. It has a role to play in treating its employees well, preserving the environment, developing sound corporate governance, supporting philanthropy, fostering human rights, respecting cultural differences and helping to promote fair trade, among others. All are meant to have a positive impact on the communities, cultures, societies and environments in which companies operate.

These efforts should also benefit a company's various stakeholders, who comprise all or some of the following: customers, employees, executives, non-executive board members, investors, lenders, vendors, suppliers, governments, NGOs, local communities, environmentalists, charities, indigenous people, foundations, religious groups and cultural organisations. 
termes figurant dans les rubriques principales ou secondaires, nous avons pu constater que, selon les entreprises, certains termes pouvaient se retrouver tour à tour en position d'hypéronymes ou d'hyponymes. Il nous a semblé que ces choix pouvaient être porteurs de sens et nous invitaient à prendre en compte les nuances possibles. Si nous nous reportons au tableau en annexe 2, nous constatons que le terme qui revient le plus fréquemment dans les rubriques principales des sites en ligne des entreprises du FTSE4Good UK 50 est Corporate Responsibility (26 entreprises sur 50), devant Corporate Social Responsibility (11 entreprises); en revanche, le tableau en annexe 1 montre que les entreprises du DJSI US 50 sont plus partagées, dans l'ordre décroissant, entre Corporate Citizenship et Corporate Responsibility. On remarque également, en annexe 3 , que le type de rapport privilégié par les entreprises des deux indices est également le Corporate Responsibility Report, devant le Corporate Social Responsibility Report.

\section{L'implicite au niveau des termes}

Il convient maintenant de nous intéresser plus particulièrement aux trois rubriques mises en avant par les entreprises pour communiquer avec le public qui visite leur site. Conformément à notre approche de l'implicite, il nous faut souligner que les «étiquettes» ainsi choisies par les entreprises semblent tenir pour acquises trois idées que nous souhaiterions analyser plus en détail: la première est que les entreprises ont des responsabilités, et il nous faudra définir quelles sont ces responsabilités; la seconde suggère que ces responsabilités peuvent être d'ordre social; enfin, la troisième pose le principe de l'entreprise citoyenne et ce présupposé demande réflexion.

\section{Corporate Responsibility}

Quelles sont donc les responsabilités de l'entreprise? Pour ceux qui maintiennent que «The business of business is business», autrement dit, que la vocation première d'une entreprise est de faire des affaires en réalisant des bénéfices, la responsabilité de l'entreprise est donc de créer de la richesse en vendant des produits ou services qui sont de nature à satisfaire les clients, de sorte que ces derniers seront fidèles et assureront un revenu qui permettra de contenter les actionnaires, source de capital pour l'entreprise. La qualité des produits et services est toutefois soumise à la qualité du travail fourni par les travailleurs ou employés, et la responsabilité de l'entreprise est donc également de s'assurer le concours des meilleurs employés, de savoir les garder et de faire en sorte qu'ils donnent le meilleur d'eux-mêmes. Ces responsabilités se confondent 
avec l'intérêt des différentes parties prenantes de l'entreprise et la théorie moderne en matière de gestion a bien intégré ceci. Les termes qui illustrent le mieux ces pratiques dans les tableaux en annexe 1 et 2 sont ceux qui évoquent les relations des entreprises avec leurs différents partenaires et leur actionnariat, ainsi que la gestion générale de l'entreprise : management, governance, workplace, employees, customers, stakebolder engagement, shareholder information, supply chain responsibility, partners, people, etc. Le sigle CR pourrait tour à tour être explicité par corporate responsibility, corporate reputation et corporate relationships.

Ces responsabilités, on en conviendra aisément, entretiennent forcément des liens avec des considérations sociales (employee well-being, par exemple, ou labour practices). Ceci nous conduit à poser la question de ce qu'il convient de mettre dans l'enveloppe «social».

\section{Corporate Social Responsibility}

Certains diront que si responsabilité sociale de l'entreprise il y a, elle doit se limiter à la création de richesse et d'emploi, et que tous les autres objectifs sociaux doivent incomber à l'État ou à d'autres organismes. À ce niveau, il y aurait peu de différence avec ce que nous avons décrit des responsabilités de l'entreprise, puisque, pour créer de la richesse, l'entreprise est dépendante de ses différents partenaires. Selon cette approche, l'ajout ou le retrait de l'adjectif social ne serait pas significatif. Toutefois, la question qui se pose semble plutôt relever de la dose de «social» qui peut varier: cela supposerait, selon les entreprises, une responsabilité sociale a minima et une responsabilité sociale plus étendue qui engloberait tout ce qui peut faire du bien, faire le bien autour de l'entreprise en termes de dons, de volontariat, de philanthropie, et de programmes pour aider les communautés en difficulté. Les termes clés qui portent ce message dans les intitulés des rubriques sont Charities \& Fundraising, Grants, Community Programmes, Corporate Giving, Philanthropy, HIVIAids initiatives, Social Commitment, Education, Employee Engagement.

Pour Waddock (2004), la responsabilité sociale s'envisagerait comme un tronc dont partiraient plusieurs branches: corporate responsibility(ies), corporate citizenship, stakeholder relationships, corporate social performance. Frederick (1987), en son temps, avait explicité le sigle CSR en Corporate Social Responsibility, Corporate Social Responsiveness, et Corporate Social Rectitude. Même si cette répartition n'a pas été conservée depuis, les notions qu'elle véhicule sont toujours présentes dans nos termes clés: compliance, ethics, buman rights, code of conduct, etc. Par la suite, Frederick (1998) a également suggéré un nouveau développement du sigle CSR en Cosmos, Science et Religion. Enfin, à partir du terme compliance, on peut entrevoir une autre distinction entre, d'une part, une RSE imposée et 
une RSE volontaire. Dans le cas de RSE volontaire, une entreprise peut très bien choisir de se conformer à des normes qu'elle a pu s'imposer, parce qu'elles correspondent à des valeurs qui sont les siennes depuis longtemps: on trouve d'ailleurs dans les rubriques retenues our (core) values, our vision, ou encore our commitment. Une entreprise peut également décider de se fonder sur des critères et des textes officiels qui servent de cadre à sa philosophie ou sa politique en matière de responsabilité sociale. De proactive, la RSE peut également être réactive, en réponse à des pressions exercées par certains groupes d'actionnaires, de consommateurs, ou d'ONG, voire imposée et dictée par la nécessité de respecter la loi en termes de droit du travail, égalité des chances, absence de discrimination, absence de corruption, etc. Senge (1994:219) parle de «genuine compliance» (démarche sincère et volontaire pour respecter les règles et même faire plus), de formal compliance (en référence au strict respect des règles) et ajoute une dernière catégorie grudging compliance, (respect de la loi sans enthousiasme ni bonne humeur). Il est évidemment difficile, à ce stade, de faire la part des choses quant aux motivations réelles des entreprises: philosophie particulière, ou mesures de prudence pour éviter de voir leur réputation ternie. Il nous faudra déceler des indices plus précis dans notre analyse de l'implicite au niveau des discours.

Si le tableau sur les rapports spéciaux des entreprises en annexe 3 ne comprend pas les noms de toutes les entreprises des deux indices, c'est aussi parce que la RSE peut faire l'objet d'un compte rendu incorporé au rapport annuel classique des entreprises, sans donner lieu à un rapport séparé; certaines entreprises mettent en avant l'idée que la responsabilité sociale est diffuse et concerne tous les niveaux de leur organisation, alors que pour d'autres entreprises, elle est plus particulièrement orientée vers certains secteurs. Il ne faudrait pas en conclure pour autant que les entreprises qui établissent un rapport séparé limitent leurs efforts en matière de responsabilité sociale à un simple gage de bonne volonté. Elles peuvent simplement avoir fait le choix de communiquer différemment avec le public. Matten \& Moon (2004) expliquent également que l'on peut parler de RSE explicite et de RSE implicite. Selon eux, la RSE explicite est volontaire; elle n'est pas institutionnalisée par des normes extérieures. Elle fera nécessairement l'objet d'une information spéciale. En revanche, la RSE implicite consiste à simplement obéir à des normes claires et précises imposées par la société ou le gouvernement; dans ce cas, l'entreprise n'a pas le choix, et n'a pas non plus besoin de communiquer par le biais d'un programme bien particulier. Elle ne fait qu'obéir aux règles. On se rend compte que sous une même appellation peuvent se cacher des conceptions bien différentes, qu'il nous faudra élucider en analysant les discours. 


\section{Corporate Citizenship}

Notre dernier terme phare est Corporate Citizenship. Mis en avant, comme simple «étiquette» il semble s'imposer de lui-même, alors que les présupposés sont porteurs de sens. Nous rappellerons la métonymie de notre intitulé «le discours des entreprises » pour évoquer la personnification de l'entreprise, mais, avec corporate citizenship, nous passons à la métaphore, non neutre, qui nécessite une analyse des sèmes empruntés à la sphère politique et aux attributs du citoyen. Il convient, en effet, de poser la question de la pertinence de cette métaphore et de s'interroger sur les notions qu'elle suggère et le message qu'elle véhicule. Pour Moon et al (2003), cette métaphore empruntée au domaine de la politique doit être replacée dans un contexte plus large. En effet, d'autres concepts, tels que la démocratie, ou la souveraineté, par exemple, ont fait leur entrée dans le monde des entreprises: ils citent «stakeholder democracy» ou encore «consumer sovereignty». Le choix du domaine source - la politique - est intéressant à plusieurs égards. On notera que les notions empruntées sont évolutives: le débat politique a fait évoluer la notion de citoyenneté au fil du temps avec le droit de vote des femmes, la prise en compte du multiculturalisme, des changements dans les institutions. La notion est donc complexe, et, une fois adaptée au monde des entreprises, il n'appartient pas aux seules entreprises de la définir; elle est revisitée de multiples manières par les différentes parties prenantes, soit pour la défendre, soit pour la critiquer. En quoi la citoyenneté s'applique-t-elle aux entreprises et est-il si évident que les entreprises puissent être considérées comme citoyennes, et, en l'occurrence, s'efforcent d'être de bonnes citoyennes? L'analogie avec les citoyens ne semble pas valable si l'on s'en tient aux droits essentiels du citoyen, en termes de statut politique et de légalité, à être protégés de l'arbitraire et de l'oppression, à être traités dignement, et à exprimer leur avis en élisant des représentants qui auront pour mission de diriger le pays. En revanche, si l'on privilégie d'autres sèmes, on peut ouvrir des perspectives nouvelles en ce qui concerne les relations entre les entreprises et la société, et, cette foisci, c'est le sème de la participation qui se trouve activé. Déjà en 1993, Drucker, en précurseur que l'on sait, privilégiait cette approche de la citoyenneté pour l'entreprise:

$\mathrm{Au}$ sens politique, citoyenneté signifie engagement actif, responsabilité. Cela veut dire qu'on contribue à changer les choses dans le groupe, la société et le pays $^{14}$. (cité par Andriof \& McIntosh 2001 : 14)

14. As a political term, citizenship means active commitment; it means responsibility. It means making a difference in one's community, one's society, and one's country 
Le terme commitment fait bien partie des termes phares choisis par les entreprises sur leurs sites en ligne. La citoyenneté renvoie alors à la notion de respect du bien public, de vertu civique, de volonté de s'engager pour ou avec la communauté, de respect des lois, de discipline quant il s'agit de payer sa part des impôts, etc. On conçoit mieux, dans cette optique, que la notion de citoyenneté puisse s'appliquer à l'entreprise. Non seulement l'entreprise a des responsabilités en interne, et doit appliquer en son sein le principe de citoyenneté délibérative en ouvrant le dialogue avec toutes les parties prenantes, mais elle a également des responsabilités en externe, vis-à-vis de la société. Selon le degré de développement des systèmes politiques des pays dans lesquelles les entreprises sont présentes, la participation pourra se traduire par un partage des responsabilités, soit avec le gouvernement en place, dans la mesure où il ne peut faire face sur tous les fronts pour résoudre les problèmes de société, soit sous la forme de partenariat avec des ONG ou d'autres organismes à but lucratif ou non. Dans d'autres cas, les entreprises se substitueront au gouvernement qui manquerait à son devoir de protéger ses propres citoyens. Respecter et contribuer au bien public peut concerner aussi bien la sauvegarde de l'environnement, la protection de la santé, l'alphabétisation ou la formation de manière plus générale, que la défense de droits bafoués, ou l'aide à des catégories de personnes oubliées par les gouvernements ou les autorités locales. Les situations peuvent donc forcer le rôle participatif de l'entreprise à évoluer, l'entreprise devenant quasiment un groupe de pression pour faire avancer une cause, ou contribuer au mieux-être des hommes. La question est d'ailleurs de savoir si l'entreprise peut ou doit prendre le relais ou se substituer aux politiques pour assurer, en interne comme en externe, les droits et la protection des employés et des autres citoyens. La notion de Global citizenship laisserait entendre que les multinationales ont un devoir (pouvoir) envers la planète qui dépasse les limites nationales et les place au-dessus du niveau des gouvernements individuels.

Il y a donc, là aussi, de nombreuses interprétations possibles pour les quelques termes-clés évoqués, et de nombreux embranchements dans la carte notionnelle révélée par les tableaux 1,2 et 3 . La répétition de ces termes n'est pas sans effet sur les esprits, comme le souligne l'analyse du philosophe Alain Etchegoyen ${ }^{15}$ sur le pouvoir des mots:

De l'expression écrite apparemment plus contrôlée à l'expression orale moins maîtrisée, des mots s'imposent à notre conscience que nous prenons ici et là : extraits de nos lectures, repris de notre éducation, captés dans l'environnement immédiat, ils nous échappent en partie. Notre époque est caractérisée par une

15. Site en ligne consulté le 4 mars 2007 <www.institut-expression.com> 
multiplicité de flux et d'échanges qui nous privent d'une concentration sur les mots que nous utilisons et d'une suspension de la conscience pour les utiliser à bon escient. C'est pourquoi, très souvent, le processus s'inverse: loin de dire ce que nous pensons, nous pensons ce que nous disons; en effet, nous ne pensons pas en dehors des mots. Comme nous ne prenons plus le temps de nous arrêter sur leur sens, de disséquer leur contenu, d'analyser leurs usages, les mots prennent le pouvoir sur notre pensée.

Il convenait donc d'attirer l'attention du lecteur sur la variété des interprétations, comme sur le fait qu'associer «social» à «Corporate Responsibility», ou encore de lui préférer «Corporate Citizenship» peut ne pas être anodin.

\section{L'implicite au niveau du discours}

Si l'observation des sites en ligne des grandes entreprises qui nous intéressent dans cette étude nous renseigne par la simple consultation des notions mises en avant dans les onglets et les menus déroulants, il va de soi que les conclusions qui peuvent déjà en être tirées demandent à être complétées par l'analyse des discours officiels des responsables de ces entreprises. Il nous a semblé que la meilleure façon de connaître la position des entreprises et d'analyser ce qui est implicite dans le discours était de consulter les rapports spéciaux élaborés par les entreprises pour communiquer sur leurs actions en matière de responsabilité sociale et environnementale, et de nous concentrer sur les textes signés par les dirigeants et qui introduisent ces rapports. Comme l'indique le tableau en annexe 3, du côté FTSE4Good, la quasi-totalité des entreprises (48 sur 50) ont fourni un rapport spécifique sur le sujet, et 34 des 48 rapports comprennent un message des dirigeants en guise d'introduction. Pour le DJSI, les rapports particuliers sont moins nombreux (32 entreprises sur 50 ) et seuls 29 rapports sont introduits par un texte des dirigeants. Selon les cas, ces rapports sont ceux de 2005 ou de 2006. Le tableau en annexe 3 met également en évidence les préoccupations principales des rapports à travers le choix de la catégorie de rapport arrêté par les entreprises. Ce choix est cohérent au regard des rubriques principales sélectionnées par les entreprises. Pour le FTSE4Good, la catégorie «Corporate Responsibility Report» arrive nettement en tête, alors que les entreprises constituant le DJSI sont partagées entre «Corporate Responsibility Reports», «Corporate Social Responsibility Reports» et «Sustainability Reports». Un regard sur le tableau en annexe 4 est instructif, car les titres supplémentaires que certaines des entreprises donnent à leur rapports viennent en écho à certains des points que nous allons être amenée à discuter. 


\section{Caractéristiques génériques et ancrage énonciatif}

Nous ne pouvons envisager l'implicite dans ces discours qu'à partir de leur contenu explicite et nous les avons donc abordés dans leur forme et dans leur fond. Bien que les textes introductifs ne suivent pas tous un plan uniforme, et reflètent sans doute la personnalité de leurs auteurs, ils présentent néanmoins des traits communs qui permettent de les considérer comme un genre particulier. En effet, ils sont tous rédigés par des dirigeants de l'entreprise ou par des directeurs de la division RSE. Le public visé par ces introductions est un public large, qui peut être celui des parties prenantes, mais aussi les analystes, gérants de fonds éthiques, agences de notation ou, plus généralement, toutes les personnes intéressées par les engagements et les actions des entreprises, dans un contexte général de prise de conscience des problèmes liés à l'environnement et à la société. Pour la grande majorité des introductions, qui varient en taille depuis l'équivalent d'une moitié de page jusqu'à cinq pages, le discours comporte une entrée en matière, qui peut rappeler l'histoire de l'entreprise, sa philosophie, des principes de base ou des généralités, ou encore faire allusion à la fin d'un mandat ou à l'arrivée de nouveaux responsables, puis un bilan rapide de l'année passée et l'annonce de projets, précisés parfois par un sous-titre (Looking Ahead, The Way Ahead) avec pour souci l'insistance sur la cohérence de l'engagement et la volonté de toujours faire mieux. Cette progression est illustrée par certains des titres qui figurent en annexe 4. C'est le cas pour Cadbury Schweppes (Living our Values: Yesterday, Today, Tomorrow), Prudential (Sowing Seeds, Growing Futures), PespsiCo (Tomorrow > Today), HSBC (Future History) et Vodafone (We said, We Have, We will). Ce dernier titre, qui illustre bien les choix des différents temps qui sont aussi ceux des divers mouvements des textes, souligne également une idée largement répandue dans les introductions, à savoir qu'il faut plus que des paroles pour convaincre et que les engagements pris doivent être respectés.

Toutefois, la première préoccupation est bien de faire adhérer les lecteurs à des idées avant de passer à la mise en pratique. Afin de convaincre leur large public et de gagner ou conserver sa confiance, les dirigeants ont donc recours aux techniques classiques de la rhétorique selon Aristote: ethos, logos et pathos. L'ethos discursif est ici lié à l'image que l'entreprise entend donner d'elle-même et à l'image de ses dirigeants qui signent leurs messages de leur nom accompagné de leur position dans la hiérarchie. Leur implication dans ce qu'ils avancent est clairement portée par le choix du pronom «I», que l'on note soit au début, soit à la fin du texte ou dans les deux cas parfois, et qui nous rappelle que nous sommes en présence de textes qui font autorité et ne sont pas neutres. Le recours au «je» marque toujours un moment fort du message. Les 
quelques exemples suivants illustrent les interventions personnelles des dirigeants qui visent à exprimer leur empathie ou à affirmer une prise de position personnelle pour donner plus de poids aux choix faits par l'entreprise :

Je suis particulièrement fier des hommes et des femmes de Chevron qui ont fait preuve d'un courage et d'un engagement extrêmes dans leurs réactions aux catastrophes naturelles de l'année dernière au cours desquelles bon nombre d'entre eux, comme tant d'autres, ont tout perdu ${ }^{16}$. (Chevron)

Je crois que notre réussite en tant qu'entreprise et notre capacité à exceller en terme de durabilité sous toutes ses formes vont de pair $^{17}$. (Baxter)

Aviva a pris le parti de la RSE et je suis convaincu de la valeur que cela représente pour notre entreprise et toutes ses parties prenantes. Cela consolide les produits que nous offrons à nos clients, améliore la qualité du travail de nos employés, bénéficie à la société en général et crée de la valeur pour nos actionnaires ${ }^{18}$. (Aviva)

Les auteurs présentent le plus souvent leur texte introductif comme un message, parfois comme une lettre («Dear Stakebolders ), tout en sachant que leur texte sera lu en ligne par qui le souhaitera. Dans deux cas, l'introduction relève d'une mise en scène plus travaillée, puisqu'il s'agit d'un entretien qui permet au responsable, dans une série de questions-réponses, de devancer les objections et les critiques éventuelles.

Pour la linguistique de l'énonciation, la notion d'ethos trouve un écho dans l'idée que les différents partenaires d'un échange se font l'un de l'autre. Il s'établit alors un pacte de lecture entre l'auteur et le lecteur. Ainsi, les auteurs des introductions sont conscients que non seulement, ils s'adressent aux parties prenantes (employés, clients, fournisseurs, partenaires, actionnaires), mais également à un cercle plus large: les investisseurs, les gérants de fonds communs de placement, les gouvernements, les ONG, les journalistes, les militants de différentes causes, les anti-mondialistes, souvent critiques à l'égard du capitalisme et de la recherche du profit, les gouvernements. Il s'agit donc d'imaginer un public dont l'opinion est soit favorable, soit neutre, soit cynique.

16. I am particularly proud of the men and women of Chevron who showed tremendous courage and commitment in their response to last year's natural disasters when many of them, like so many others, lost everything. (Dave O' Reilly, Chairman and CEO of Chevron Corporation)

17. I believe our success as a business and our ability to excel across all aspects of sustainability go hand in hand (Robert L. Parkinson, Jr., Chairman and CEO, Baxter)

18. Aviva is committed to CSR and I am convinced of the value it brings to our business and to all its stakeholders. It reinforces the products we offer to our customers, enhances the performance of our staff, promotes social benefits and delivers value to our shareholders. (Richard Harvey, Group Chief executive, Aviva) 
Pour relever ce défi, il leur faut trouver des arguments pour convaincre en faisant tour à tour appel à la raison, à l'émotion, aux sentiments, au sens de l'éthique; de ce fait, logos et pathos s'entremêlent dans ces textes. Le logos est, en l'occurrence, caractérisé par la démonstration logique selon laquelle ce qui est bon pour la communauté, la société et l'environnement est aussi bon pour l'entreprise:

Chez Diageo, nous ne mesurons pas la réussite en termes de retours financiers. Nous avons une approche globale des résultats de notre entreprise, qui inclut nos progrès en matière d'engagements sociétaux et environnementaux. Nous ne pensons pas qu'il faille choisir entre réussir au plan financier et réussir à remplir notre rôle d'entreprise citoyenne. Créer de la valeur et vivre selon nos valeurs sont deux conditions essentielles si Diageo veut continuer à prospérer et à se développer en tant qu'entreprise réellement durable ${ }^{19}$. (Diageo)

Contrairement à l'idée reçue, les considérations commerciales d'une entreprise et le respect de la planète et des autres citoyens ne sont pas contradictoires. En fait, nous pensons que l'histoire de Schlumberger montre que ces aspects sont absolument compatibles ${ }^{20}$. (Schlumberger)

Nous avons toujours soutenu que la première responsabilité sociale d'une entreprise est de réussir. La réussite nous permet d'investir dans de nouveaux produits et services pour nos clients. Elle nous permet de verser les dividendes qui constituent une part tellement importante des économies à long terme et des plans d'épargne retraite de nos actionnaires. Elle nous permet de contribuer aux services publics en nous acquittant de nos impôts envers les gouvernements. Elle crée des emplois pour nos collègues et fournisseurs. Cela ne veut pas dire que le souci de réaliser des bénéfices doit conduire une entreprise à négliger les obligations sociétales plus larges qui lui incombent. Loin d'être incompatibles, réussite durable et bonne conduite sont inextricablement liées ${ }^{21}$. (HSBC)

19. At Diageo, we don't measure success solely in terms of financial return. We take a holistic view of our company's performance, including our progress against social and environmental policy commitments. We don't believe we have to choose between financial success and being a good corporate citizen. Creating value and living our values are both essential requirements for the continuing prosperity of Diageo and its development as a truly sustainable business. (Paul S. Walsh, Chief Executive Chaiman, Diageo)

20. Contrary to popular opinion, commercial enterprise and respect for our planet and fellow citizens are not necessarily contradictory. Indeed, we believe the history of Schlumberger proves that they can be entirely compatible. (Andrew Gould, Chairman \& CEO, Schlumberger)

21. We have always maintained that a company's first social responsibility is to be successful. Success allows us to invest in new products and services for our customers. It enables us to pay the dividends which form such an important part of the long-term savings and pension plans of our shareholders. It allows us to contribute to public services through the taxes we pay to governments. It creates jobs for our colleagues and suppliers. This is not to say that profits should be pursued without concern for a company's wider social obligations. Far from being incompatible, long-term success and good corporate behaviour are linked inextricably. (Sir John Bond, Group Chairman, HSBC) 
On notera dans les exemples ci-dessus l'utilisation du pronom «nous» («we / us»), le plus fréquent dans ces textes, et relayé parfois par l'adjectif possessif «notre / nos» (« our»). Il convient d'en souligner les différentes valeurs. Ici, il renvoie à une entité abstraite, à un locuteur absent, qui serait la direction, le Board of Directors, ou l'entreprise elle-même, voire le groupe. Dans d'autres cas, indéniablement, «we» permet de susciter l'adhésion des parties prenantes et de les inclure dans les décisions et actions: « we will continue together to make sustainability integral to our success $\gg$ (Procter and Gamble). Il sert également à signifier à l'ensemble des collaborateurs que les entreprises sont de grandes familles, des groupes où tous ont leur place et à leur renvoyer une image positive de l'entreprise et de ses actions. À cet égard, certains titres de rapports sont évocateurs, comme «My Starbucks» ou «Your M\&S». Chacun des collaborateurs de l'entreprise, à un titre ou à un autre, peut alors se sentir directement impliqué, comme si le texte lui était directement adressé: il peut s'identifier complètement à l'entreprise qui devient son entreprise, ou s'identifier au groupe (nous, c'est-à-dire vous et moi, sommes concernés, engagés, et nous partageons les mêmes valeurs et poursuivons les mêmes objectifs). Pour les lecteurs extérieurs, «nous / we» suscitera l'adhésion à ce qui peut être présenté comme une vérité générale et sans appel au niveau des commentaires sur le rôle de l'entreprise moderne (vous et moi sommes d'accord pour reconnaître ceci), ou il montrera que toutes les parties prenantes de l'entreprise sont unies dans un même but (dans cette entreprise, nous oeuvrons tous dans la même direction).

Également présent dans le discours, le pathos est convoqué pour produire, selon les cas, trois effets, illustrés ci-dessous. En premier lieu, il suscite l'adhésion à l'idée de soutenir des causes louables ou d'aider les défavorisés en jouant sur la fibre sentimentale; en second lieu, il pose l'entreprise en «héros positif», capable de relever de grands défis économiques et humanitaires; enfin, il permet à l'auteur de montrer qu'il est capable de compassion, d'humanité et, même, refusant toute complaisance, d'humilité, lorsqu'il reconnaît publiquement les erreurs, et s'engage à les corriger :

J'ai été particulièrement fier des efforts consentis par nos gens pour venir en aide aux communautés locales et réparer l'infrastructure indispensable pour l'alimentation en énergie après le tsunami en Asie, les séismes au Pakistan et en Inde, et les ouragans dans le Golfe du Mexique aux États-Unis. Nous avons été la première grande entreprise à reprendre nos activités à la Nouvelle-Orléans après les ouragans, et, pour moi, ceci témoigne notre profond engagement envers les communautés dans lesquelles nous intervenons ${ }^{22}$. (Shell)

22. I was particularly proud of our people's efforts to help local communities and repair vital energy infrastructure after the Asian tsunami, the earthquakes in Pakistan and India, and the hurricanes in the US Gulf of Mexico. Being the first major company to 
Douze de nos employés ont perdu la vie, cinq pour Otis et sept chez UTC, dans la branche incendie et sécurité. Je me penche toujours personnellement sur chaque accident mortel, en relation avec l'ensemble des personnes en position d'autorité à tous les niveaux afin de déterminer les faits à l'origine des accidents et de modifier les pratiques et l'équipement en fonction des accidents ${ }^{23}$. (United technologies Corporation $)^{23}$

Il convient également de noter que les dirigeants, comme l'a très justement souligné Igalens (2006), content une histoire, celle de l'entreprise, plus qu'ils ne rendent des comptes. La part de narration est effectivement beaucoup plus importante que les quelques chiffres qui sont mentionnés en passant et qui seront développés dans la partie comptable du rapport. Il faut comprendre ici qu'ils insistent sur la tradition de l'entreprise, ses valeurs, sa philosophie, ses progrès, difficultés, et engagements pour mieux ancrer les mesures récentes et à venir dans la vie de l'entreprise.

\section{De la forme au fond}

En dehors des effets de rhétorique, les choix lexicaux sont également porteurs de message. Une extraction systématique des substantifs les plus souvent rencontrés ${ }^{24}$ met en évidence deux listes assez semblables des 10 substantifs les plus présents dans le discours pour les deux indices, mais on note quelques différences dans l'ordre de fréquence décroissant. Si business et company arrivent à égalité en tête de liste dans les deux cas, ce qui s'explique logiquement puisqu'il s'agit de présenter l'activité de l'entreprise, on note que sharebolder n'est qu'en dernière position pour les entreprises du FTSE, et absent de la liste pour le DJSI. En revanche, stakebolder occupe le même rang dans les deux indices, relativement bas dans le tableau. Corporate Responsibility et sustainability ont été extraits de la liste car il est évident que leur poids n'est pas significatif dans la mesure où les textes introduisent les rapports qui portent ces mêmes noms. La fréquence d'utilisation de customer est nettement moindre dans le classement du DJSI par rapport à la deuxième position qui lui est accordée pour le FTSE. Community, employee, commitment et people sont à peu près également représentés, mais une différence marquée est établie

return its operations to New Orleans after the hurricanes exemplifies for me our deep commitment to the communities where we operate. (Jeroen van der Veer, Chief Executive, Royal Dutch Shell)

23. Twelve employees lost their lives, five at Otis and seven at UTC Fire \& security. I review each fatality personally with the entire operating chain of command to determine root causes and change practices and equipment accordingly. (George David, Chairman and CEO, United Technologies Corporation)

24. Cette extraction a été faite à l'aide de l'extracteur en ligne Termostat. 
pour value/values, beaucoup plus présents dans le FTSE4Good, que pour le DJSI. Compliance est absent du FTSE, et traduit peut-être le fait que les entreprises américaines opèrent dans un contexte où le risque de poursuites en justice est plus pressant que pour leurs homologues au Royaume-Uni.

Au niveau des verbes, dont les divers temps traduisent bien le lien entre passé, présent et avenir, et marquent ainsi la cohérence de la démarche, les choix sont riches d'enseignements. Les verbes le plus souvent rencontrés renvoient à l'engagement moral ou réel de l'entreprise (aim, believe, commit, comply, plan), aux actions réellement menées et à leurs effets (benefit, collaborate, contribute, create, donate, help, implement, improve, launch a campaign, meet needs, offer, provide, put in place, reduce our water usage, serve, share, etc.), au désir de devancer la contradiction et de forcer le consensus (recognize, know) et au souci d'exprimer des intentions fermes (want). On note enfin quelques verbes à valeur illocutoire (Searle 1972), comme commission, instruct, ou encore order et thank qui traduisent le passage à l'action.

Plus significatifs, toutefois, sont des termes qui ne reviennent peutêtre qu'une seule fois dans chaque introduction, mais qui sont vecteurs d'idées importantes et sont riches en information sur la position des entreprises. Tel est le cas du verbe «embed», qui insiste sur une conception répandue parmi ces entreprises que la RSE doit être inhérente à toutes les activités de l'entreprise, parce qu'ancrée dans ses valeurs et que, si elle est artificiellement plaquée a posteriori, elle n'est ni efficace, ni crédible:

Je pense que le RSE est réellement importante quand elle s'intègre à l'action. Il convient de l'introduire dans l'ADN de l'entreprise afin qu'elle devienne intuitive. Je sais qu'elle relève d'une science, mais c'est aussi un art. Elle participe à la fois de la tête et du cœur, mais n'est ni complètement l'un, ni complètement l'autre. [...] Nous ne gérons pas nos activités pour ensuite nous dire «maintenant, il nous faut aussi penser à la RSE». Elle est inhérente à l'entreprise et relève du simple bon sens. Tous les éléments de la RSE traduisent naturellement la façon dont on veut vivre, travailler, être traité et se développer ${ }^{25}$. (British Telecom)

Les conseils d'administration qui considèrent que la responsabilité des entreprises vient se plaquer sur le reste des activités risquent de ne remplir leurs obli-

25. I believe that CSR is truly important as a component of what you are doing. You need to bring it into your DNA, and you need to make it an intuition. I know there is a science to this. But is it also an art. It is head and heart but not just head and not just heart. [...] We do not do our business and then think "now we must also think about CSR. It is embedded in what is common sense for the company. All the elements of CSR are a natural way of how you want to live, to do business, to be treated and to develop. (Ben Verwaayen, Chief Executive, British Telecom) 
gations ni envers leurs actionnaires ni envers quiconque ${ }^{26}$. (Alliance \& Leicester) Nous ne croyons pas qu'il soit possible d'être «responsable à moitié » ou de sélectionner les secteurs où il sied d'être responsable27. (Vodafone)

La responsabilité sociale de l'entreprise ne passe pas en second dans notre entreprise. Elle est au centre de nos actions ${ }^{28}$. (Time Warner)

Cette idée louable et exprimée haut et fort, selon laquelle il convient d'ancrer la $\mathrm{R}(\mathrm{S}) \mathrm{E}$ dans les valeurs de l'entreprise et de l'intégrer à toutes les activités de tous les secteurs de l'entreprise, demande réflexion. En effet, comme le soulignent bon nombre de responsables, si la RSE est inscrite dans les gènes de l'entreprise, est-il besoin de lui donner un nom particulier? Si l'entreprise souhaite durer, elle se doit de réaliser des bénéfices, et, pour ce faire, elle ne peut se permettre de mal se conduire envers tous ses partenaires, de renier ses engagements ou de voir sa réputation flétrie et la confiance des parties prenantes perdue. Le simple fait de poursuivre ses intérêts contribue à l'intérêt commun. En ce sens, on pourrait avancer que le premier défenseur de la RSE est Adam Smith (1776) et rappeler qu'intérêt personnel (self-interest) ne saurait se confondre avec égoïsme (selfishness):

Chaque individu met sans cesse tous ses efforts à chercher, pour tout le capital dont il peut disposer, l'emploi le plus avantageux. En général, il est vrai, il n'a pas pour intention de promouvoir l'intérêt général, et il ignore dans quelle mesure il le promeut ... c'est son propre bénéfice qu'il a en vue. En cela, comme dans beaucoup d'autres cas, il est conduit par une main invisible à remplir une fin qui n'entre nullement dans ses intentions. Tout en ne cherchant que son intérêt personnel, il travaille souvent d'une manière bien plus efficace pour l'intérêt de la société, que s'il avait réellement pour but d'y travailler. [...] Ce n'est pas de la bienveillance du boucher, du brasseur ou du boulanger que nous attendons notre dîner, mais de leur souci de leur intérêt propre. Nous faisons appel non pas à leur humanité mais à leur amour pour eux-mêmes, et ne leur parlons jamais de nos propres besoins, mais de leurs avantages ${ }^{29}$.

26. Boards that treat corporate responsibility as a bolt-on, risk failing to fulfil their obligations to both shareholders and others. (Sir Derek Higgs, Chairman, Alliance \& Leicester)

27. We do not believe that you can be 'half responsible' or pick and choose the convenient areas to be responsible. (Arun Sarin, Chief Executive,Vodafone)

28. Corporate Social responsibility is not an afterthought at our company. It is central to what we do. (Richard D. Parsons, Chairman and CEO, Time Warner)

29. Every individual necessarily labours to render the annual revenue of the society as great as he can. He generally, indeed, neither intends to promote the public interest, nor knows how much he is promoting it [...] he intends only his own gain, and he is in this, as in many other cases, led by an invisible hand to promote an end which was no part of his intention. [...] By pursuing his own interest he frequently promotes that of the society more effectually than when he really intends to promote it. [...] It is not from the benevolence of the butcher, the brewer, or the baker, that we expect our dinner, but from their regard to their own interest. We address ourselves, not to their humanity but to their self-love, and never talk to them of our own necessities but of their advantages. 
Il semblerait donc qu'une partie de ce qui est compris dans l'enveloppe RSE n'est finalement qu'histoire de bonnes pratiques de l'entreprise en matière de gestion. Ne devraient donc figurer au titre de la RSE que les démarches volontaires, qui vont au-delà du strict respect des lois et de la peur des poursuites en justice. C'est d'ailleurs sur cette base de volontariat que les 10 principes du Global Compact de l'ONU (voir annexe 6) ont été proposés, en janvier 1999, à l'occasion du Forum Economique de Davos (Suisse). Et c'est dans ce même sens que l'entend la Commission Européenne (2001) qui définit le concept de RSE comme «l'intégration volontaire par les entreprises de préoccupations sociales et environnementales à leurs activités commerciales et leurs relations avec leurs parties prenantes».

Pourtant les dirigeants des entreprises troublent le message en plaçant sur le même plan les actions qui relèvent de la stricte obéissance à la loi et, comme nous l'avons expliqué à propos du contenu notionnel des termes, ce qui va au-delà du respect des règles en vigueur et des lignes directrices que l'on peut trouver dans les documents officiels mis en avant pas les dirigeants (Global UN Compact, Global Reporting Initiative (GRI), etc.). Les occurrences de «compliance» font ressortir que la notion s'applique dans les deux cas, ce qui peut créer une certaine ambiguïté car respecter les règles que l'on a fixées pour soi-même relève d'une autre démarche. Entre rendre des comptes parce qu'on est obligé de le faire et rendre des comptes parce que cela correspond à un désir personnel de clarté et de transparence, il y a, de toute évidence, une différence appréciable.

Au chapitre des démarches volontaires, s'inscrivent les dons et la démarche philanthropique: derrière le discours des dirigeants qui félicitent leurs employés de s'impliquer dans les œuvres charitables en donnant de leur temps, et donc, se félicitent eux-mêmes d'avoir su les encourager au bénévolat, la question se pose de justifier aux yeux des actionnaires le choix de consacrer une partie des bénéfices à soutenir de bonnes causes, plutôt que de réinvestir ces bénéfices ou d'augmenter les dividendes. Pour ce faire, les responsables mettent en avant ces actions charitables (lutte contre le sida, formation, instruction, etc.) comme la suite logique de la philosophie de l'entreprise, soulignant les aspects humains et moraux. On pourrait leur reprocher à deux titres de manquer de clarté à ce sujet. En premier lieu, on pourrait leur rétorquer qu'il est facile d'être généreux avec l'argent des autres, celui des actionnaires, et que la vraie philanthropie serait celle de Bill Gates, qui fait des dons à partir de son capital personnel et non en piochant dans le capital de Microsoft. Autrement dit, sous couvert d'une certaine morale, ne fontils pas preuve de désinvolture en utilisant l'argent des actionnaires comme ils l'entendent? En second lieu, car il y a évidemment une expli- 
cation au manque de réactions hostiles des actionnaires, ils ne font jamais état de manière explicite de la publicité que leur procure de telles actions qui, finalement, si elles réduisent les bénéfices d'un côté, peuvent, d'un autre côté, contribuer à l'image de marque de l'entreprise et donc à attirer de nouveaux consommateurs et clients. D'ailleurs, une entreprise responsable, soucieuse de durer, se livrera toujours à une analyse des coûts et des bénéfices, et ne prendra aucune décision qui pourrait mettre en péril l'équilibre financier. Il nous faut donc lire l'implicite entre les lignes, comprendre que les bonnes actions sont en quelque sorte un investissement sur lequel on est en droit d'attendre un retour, et appliquer à toutes les actions réalisées par les entreprises l'idée du cercle vertueux, exprimée de diverses façons dans le discours, et résumée cidessous:

Depuis toujours, chez Cisco, nous pensons que faire de bonnes actions contribue à la réussite de l'entreprise ${ }^{30}$. (Cisco)

Nos programmes ne sont pas seulement bons pour la communauté; ils sont également bons pour notre rentabilité ${ }^{31}$. (SABMiller)

\section{La teneur du message sous-jacent}

Au terme de cette analyse sur la forme et le fond, il nous reste à faire le point sur le message sous-jacent à toutes les interventions des dirigeants et sur le rôle réel des grandes entreprises dans notre monde. Pour ce faire il convient d'avoir en tête le cadre général de notre société, de ses évolutions et des événements récents qui ont marqué notre environnement. Le premier point concerne l'échec du communisme, qui ne laisse comme modèle de système économique, que le capitalisme, modèle parfois mal compris, mal intégré, et mal accepté, ce qui nourrit des rancoeurs et des contestations fortes. Le deuxième point est l'ouverture de nombreuses frontières et économies au système de libre-échange et à la concurrence, avec la multiplicité des accords à régler au niveau de l'OMC et les inévitables tensions qui peuvent en découler. Les manifestations en marge des sommets sont devenues courantes. Le troisième point concerne les scandales financiers qui ont conduit de grandes entreprises, telles Enron, Worldcom, Tyco ou Parmalat, à la faillite et qui ont marqué les esprits: de nouvelles normes comptables ont été arrêtées, obligeant à une plus grande transparence et une plus grande discipline, mais la méfiance envers le grand capital demeure. Enfin, il faut égale-

30. From our earliest days, we have believed at Cisco that doing good will always contribute to doing well. (John P. Morgridge, Chairman of the Board, Cisco)

31. Our programmes are not just good for the community, but good for profitability (Robert Fellowes, Senior non-executive Director, SABMiller) 
ment citer la mondialisation, qui oblige les entreprises à repenser leurs pratiques, à s'adapter à un nouvel environnement humain et naturel, à travailler avec des partenaires de cultures différentes, et à répondre aux exigences multiples des citoyens de divers pays. La prise de conscience des problèmes planétaires par les populations pèse également sur les décisions auxquelles les entreprises doivent faire face.

Sur ce fond de société en évolution, les entreprises ne peuvent que s'adapter pour survivre. Il ne faut pas oublier que leur existence même est liée à leur réputation, à l'image que le public a d'elles, à l'envie qu'elles arrivent à nourrir chez leurs employés de donner le meilleur d'eux-mêmes, et de garantir la qualité des produits et services, à l'envie qu'elles peuvent susciter chez les consommateurs ou clients d'acheter leurs produits et services et de leur rester fidèles, à la façon dont elles savent faire accepter leur présence dans tel ou tel endroit. Il leur faut donc obtenir ou reconquérir le droit d'exercer leurs activités. À cet égard, le titre du rapport de BHP Billiton est parlant: « License to Operate». Dans ce contexte, leur discours a pour objet de se justifier, de répondre aux attaques des opposants au capitalisme ou à la mondialisation en donnant un sens à leur mission, une raison d'être, d'agir et d'être jugées sur leurs actions. Les grandes entreprises pratiquent un capitalisme à visage humain et entendent convaincre le grand public que, si elles peuvent apporter une partie des solutions, elles ne sont en rien à l'origine des problèmes. Les mauvaises langues diront peut-être que la responsabilité sociale des entreprises est le prix qu'il leur faut payer à la vertu, mais il ne semble pas que la RSE soit adoptée par les entreprises internationales ou supranationales simplement en gage de bonne conduite. Indéniablement, avec la fin de l'État-Nation, elles ont un rôle à jouer, mais leur conception de leurs responsabilités telle qu'elle ressort de ces textes est avant tout pragmatique. N'oublions pas qu'à la différence des gouvernements, elles ne sont pas élues, et qu'elles n'ont pas reçu de mandat de la population. Elles souhaitent montrer qu'elles ne sauraient être considérées comme la panacée, comme le souligne Kingfisher: «Nous n'avons pas toutes les réponses, mais nous sommes fermement décidés à collaborer afin de rechercher des solutions et à améliorer nos résultats tous les ans en la matière ${ }^{32} \gg$.

Si elles insistent pour dire que la RSE doit être inhérente à leur stratégie d'ensemble, et intégrée à toutes leurs pratiques, elles laissent entendre qu'elles ne peuvent pas être sur tous les fronts; elles choisissent donc les secteurs où elles sont le mieux à même d'apporter une contribution réelle:

32. We don't have all the answers, but we are firmly committed to working with others to seek solutions and to improve performance every year. 
Nous avons décidé de concentrer notre attention sur les défis que nous pouvons le mieux relever grâce à notre force d'organisation, notre savoir-faire technologique et nos valeurs liées à notre culture. En conséquence, nous concentrerons nos efforts sur trois domaines distincts: les changements climatiques; santé, sécurité et environnement; et enfin l'instruction ou la formation ${ }^{33}$. (Schlumberger)

Dans le même ordre d'idées, des entreprises comme Royal Dutch Shell ou BP se préoccuperont davantage de leur impact sur l'environnement, et investiront dans la recherche d'énergies alternatives, alors que les fabricants de boissons alcoolisées s'attacheront à éduquer les gens en matière de «Responsible Drinking». De leur côté, les entreprises pharmaceutiques feront des efforts pour que tous puissent avoir accès à leurs produits et que les populations défavorisées puissent bénéficier de prix modérés. Enfin, les organismes financiers comme HSBS ou Barclays s'orienteront respectivement vers le «Social Banking» ou le «Responsible lending » et la micro finance. Il n'y a donc pas de RSE «modèle unique »; il est, au contraire, nécessaire, que les entreprises soient flexibles et s'adaptent en fonction de leurs possibilités et des besoins locaux et ponctuels. De cette manière, les entreprises répondent au mépris que certains critiques expriment à leur égard.

Les explications fournies par les entreprises quant à leur conception de leur rôle passent également par les précisions qu'elles apportent sur le choix du terme qui définit leur position:

Nous avons opté pour le terme «citoyenneté planétaire» pour décrire notre vision car il est le mieux placé pour traduire la grande diversité culturelle de nos gens, ainsi que leur volonté, individuelle et collective, de contribuer au progrès et au bien-être de ceux dont la vie est affectée par nos activités partout dans le monde. (Schlumberger) ${ }^{34}$.

Certaines entreprises sont même passées d'un terme à un autre et expliquent ce changement:

En passant de l'ancienne appellation «Rapport de citoyenneté planétaire» à «Rapport de Responsabilité de l'Entreprise», nous traduisons mieux à la fois

33. We have decided to focus our attention on the challenges where our organizational strengths, technological expertise, and cultural values will have the greatest impact. We, therefore, will focus on three distinct themes: climate change; health, safety, and environment; and education. (Andrew Gould, Chairman \& CEO, Schlumberger)

34. We have chosen the term « Global Citizenship» to describe our approach because it best reflects the great cultural diversity of our people as well as their will, individually and collectively, to contribute to the progress and well-being of those whose lives are touched by our activities around the globe. (Andrew Gould, Chairman \& CEO, Schlumberger) 
notre vision des affaires, et nos interactions avec les nombreuses parties prenantes partout dans le monde ${ }^{35}$. (Intel)

On s'aperçoit que, comme pour le type d'actions à mener, il n'y a pas de modèle fixe, et que chaque entreprise revisite les notions pour se les approprier. Cela confirme ce que nous avons avancé quant à l'implicite au niveau des termes: les frontières sont floues et perméables entre les différentes notions et ouvertes à diverses interprétations.

Enfin, on ne peut ignorer la concurrence qui existe entre les entreprises pour conserver ou gagner des parts de marché. Cette concurrence est sous-jacente à la façon dont les entreprises communiquent sur leurs activités en matière de RSE. Si on a pu, par le passé, user du terme Greenwashing, avec sa connotation négative, il semblerait que les grandes entreprises, quel que soit leur motif réel, n'aient plus le choix et ne puissent se dérober aux pressions ambiantes, ne serait-ce que pour en tirer profit. Le message explicite est «nous sommes vertueux, respectueux des êtres, de l'environnement, des règles, etc., et nous faisons même mieux que respecter les règles»; et le message implicite est «sinon, nous perdrons du terrain face à nos adversaires plus vertueux, et nous risquons même de disparaître». Ceci confirme que ce n'est pas tant l'entreprise qui a changé - car son but est bien toujours de réaliser des bénéfices et de créer de la richesse - que la société et le monde, qui la forcent à s'adapter pour répondre aux exigences nouvelles, et tenir compte des divers groupes de pressions qui pourraient affecter ses résultats et mettre en danger sa survie. En réponse, les grandes entreprises internationales peuvent également, grâce à leur taille et leur influence, aussi se poser en moteur du changement, comme le suggèrent les titres «Make it Happen», «We create Possibilities», «Powerful Opportunities» et «Changing Lives».

Au terme de cette étude, il nous semble important de revenir sur la notion même de responsabilité pour souligner qu'elle peut s'entendre avec deux connotations différentes. La première est négative: il s'agit de la responsabilité juridique et quand on demande des comptes dans ce contexte à un responsable, on est à la recherche d'un coupable. La seconde connotation, positive, fait référence à la valeur morale, à l'enga-

35. In transitioning from the previous title of Global Citizenship Report to Corporate Responsibility Report, we better reflect both our approach to business and the way we interact with our many stakeholders around the world. (Paul S. Otellini, President and CEO, Intel). 
gement. Dans ce second sens, si l'on rend des comptes, c'est simplement pour montrer que l'on a honoré ses engagements. C'est dans ce second sens qu'il faut comprendre la responsabilité des entreprises. La RSE a une connotation positive. Elle réussit à rapprocher «valeur morale» et «valeur marchande» et à démontrer qu'il n'y a pas incompatibilité.

L'implicite, nous l'avons souligné, se situe à différents niveaux, celui des présupposés inhérents aux termes eux-mêmes, celui des interprétations possibles en fonction des connotations, et celui des implicatures, que l'on peut déduire du discours. Indéniablement, ce discours agit dans ce sens qu'il «construit un univers d'influence entre les partenaires de cet acte tendant à modifier les états intellectuels et émotionnels» (Charaudeau \& Maingueneau 2002 : 24). Il ne s'agit pas de comprendre ici l'implicite comme un simple clin d'œil ou sous-entendu, mais comme des signes que le discours envoie à différents publics ciblés. L'ensemble des discours vise à ne pas cacher le but premier de l'entreprise - réaliser des bénéfices - sous-entendant qu'il n'y a pas à rougir de ce but avoué, pour mieux convaincre de manière indirecte de la sincérité de l'entreprise qui joue la transparence et en qui, par conséquent, tous peuvent avoir confiance.

Le discours des entreprises sur leurs responsabilité, nous l'avons montré, est un discours de légitimation qui fait appel autant à la raison qu'à l'émotion. Il sous-entend que les entreprises internationales peuvent offrir une vision d'un monde nouveau, dont elles seraient les nouveaux héros en acceptant de jouer un rôle, au côté et avec d'autres partenaires, pour prendre en charge les défis mondiaux: lutte contre le réchauffement de la planète, alimentation en eau potable, en énergie, aide aux populations victimes de catastrophes naturelles ou de pandémies, participation au développement économique et social des minorités défavorisées, alphabétisation et formation, défense des droits de l'homme, aide à la recherche fondamentale pour améliorer la santé, l'hygiène, les techniques, la qualité de la vie. De toute évidence, les introductions des responsables cherchent à ancrer la RSE dans les valeurs et principes moraux de l'entreprise, et la place laissée aux faits et aux chiffres est moins importante que les arguments qui visent à convaincre du bien-fondé des décisions de l'entreprise, présentée comme compatissante et responsable. Par leur capacité à faire le bien tout en se faisant du bien, les entreprises peuvent faire bouger les mentalités de leurs parties prenantes et du public et montrer que le capitalisme et l'économie de marché ont un visage humain. Ces grandes entreprises ont autant besoin de la société que la société a besoin d'elles et c'est sans doute ce message rassurant qu'elles entendent aussi faire passer implicitement. 


\section{Bibliographie}

Andriof J. \& M. McIntosh (dir.), Perspectives on Corporate Citizenship, Sheffield, Greenleaf, 2001.

Austin J.L., Quand dire, c'est faire, Traduction française par G. Lane, Paris, Éditions du Seuil, 1970.

Carroll A.B., «The Four Faces of Corporate Citizenship», Business \& Society Review, Vol. 100-101, Journal of the Center for Business Ethics at Bentley College, 1998, p. 1-7.

Caroll A.B., «Corporate social responsibility - evolution of a definitional construct», Business \& Society, 38 (3), 1999, p. 268-295.

Charaudeau P. \& D. Maingueneau, Dictionnaire d'analyse du discours. Paris, Éditions du Seuil, 2002.

Commission Européenne. «Promouvoir un cadre européen pour la responsabilité sociale des entreprises», Livre vert COM366 Final, Bruxelles, 2001.

Cramer J., A. Van Der Heijden \& J. Jonker, «Corporate Social Responsibility/ Balancing between Thinking and Acting $\gg$, ICCSR Research Paper Series $n^{\circ} 28, \mathrm{D}$. Matten (dir.) International Center for Corporate Social Responsibility, 2004, p. 1-31.

Frederick W.C., Moving to CSR4: What to Pack for the Trip, Business \& Society, 37 (1), 1998, p. 40-59.

Garriga E., \& D. Melé, «Corporate Social Responsibility Theories: Mapping the Territory », Fournal of Business Ethics, 53 (1-2), 2004, p. 51-71.

Grice H.P. «Further notes on logic and conversation». Syntax and semantics 9: Pragmatics. Peter Cole (dir.), New York, Academic Press, 1978, p. 113-128.

Hindle T., Guide to Management Ideas, Londres, Profile Books Ltd, 2003.

Igalens J., «L'analyse du discours de la responsabilité sociale de l'entreprise à travers les rapports annuels de développement durable d'entreprises françaises du CAC $40 »$, Cahier de recherche $n^{\circ} 173$, Centre de recherche en gestion de l'université de Toulouse, 2006, p. 1-28.

Matten J. \& J. Moon «'Implicit' and 'Explicit' CSR. A conceptual framework for Understanding CSR in Europe $\gg$, ICCSR Research Paper Series $n^{\circ} 29$, D. Matten (dir.) International Center for Corporate Social Responsibility, 2004, p. 1-44.

McIntosh M, D. Leipziger, K. Jones \& G. Coleman, Corporate Citizenship: Successful Strategies for Responsible Companies, Londres, Financial Times / Pitman Publishing, 1998.

Moon J, A. Crane \& D. Matten «Can corporations be citizens? Corporate citizenship as a metaphor for business participation in society », ICCSR Research Paper Series $n^{\circ} 13$, D. Matten (dir.) International Center for Corporate Social Responsibility, 2003, p. 1-28.

Resche C., «Les mission statements des grandes entreprises cotées en Bourse: prélude à l'étude d'un genre particulier de textes dictés par plusieurs contextes», ASp n ${ }^{\circ}$ 47-48, Bordeaux: Université Victor Segalen-Bordeaux 2, 2005, p. 5-30.

Robert P., Dictionnaire alphabétique et analogique de la Langue française, t. 3, 1977

Searle J. R., Les actes de langage, trad. Fr., Paris, Hermann, 1972.

Senge P. M., The Fifth Discipline. New York, Currency Doubleday,1994.

Shore B. «Doing Well by Doing Good», The McKinsey Quarterly Nonprofit Anthology, 2001.

Swanson D. L., «Addressing a theoretical problem by reorienting the corporate social performance model», Academy of Management Review, 20 (1), 1995, p. 4364. 
Waddock S., «Parallel Universes: Companies, Academics, and the Progress of Corporate Citizenship» Business \& Society Review, Vol. 109, Journal of the Center for Business Ethics at Bentley College, 2004, p. 1-32.

Wood D. J., «Social issues in Management: Theory and Research in Corporate Social Performance», Fournal of Management 17 (2),1991, p. 383-406.

\section{ANNEXES}

\section{ANNEXE 1 - TABLEAU DES 50 ENTREPRISES COMPOSANT}

LE DOW FONES SUSTAINABILITY INDEX (US), FÉVRIER 2007

\begin{tabular}{|c|c|c|c|}
\hline Enterprises & Secteur & $\begin{array}{l}\text { Rubrique } \\
\text { principale }\end{array}$ & Sous-catégories \\
\hline Adobe Systems Inc. & Technology & $\begin{array}{l}\text { Adobe and } \\
\text { the Environment }\end{array}$ & Communities \\
\hline $\begin{array}{l}\text { Abbott } \\
\text { Laboratories }\end{array}$ & Health Care & Global Citizenship & $\begin{array}{l}\text { Ethical Business Practices, } \\
\text { Environment, Health \& } \\
\text { Safety, Philanthropy }\end{array}$ \\
\hline Aetna Inc. & Health Care & Corporate Responsibility & $\begin{array}{l}\text { Diversity, Corporate } \\
\text { Governance }\end{array}$ \\
\hline Alcoa Inc. & Basic Materials & Sustainability & $\begin{array}{l}\text { Governance, Community, } \\
\text { Environment, Health \& } \\
\text { Safety }\end{array}$ \\
\hline Allstate Corp. & $\begin{array}{l}\text { Finance, } \\
\text { Insurance }\end{array}$ & $\begin{array}{l}\text { Community } \\
\text { Commitment }\end{array}$ & CSR Report \\
\hline Amgen Inc. & Health Care & Corporate Philanthropy & $\begin{array}{l}\text { Corporate Giving, } \\
\text { Corporate Compliance }\end{array}$ \\
\hline $\begin{array}{l}\text { Applied Materials } \\
\text { Inc. }\end{array}$ & $\begin{array}{l}\text { Nanomanufacturing } \\
\text { Technology Solutions }\end{array}$ & Social Responsibility & \\
\hline $\begin{array}{l}\text { Bank of America } \\
\text { Corp. }\end{array}$ & Finance & Commitment & Community Impact Report \\
\hline $\begin{array}{l}\text { Baxter International } \\
\text { Inc. }\end{array}$ & Health Care & Sustainability Report & \\
\hline $\begin{array}{l}\text { Bristol Myers } \\
\text { Squibb Co. }\end{array}$ & Health Care & Corporate Responsibility & $\begin{array}{l}\text { Corporate Philanthropy, } \\
\text { Sustainability, } \\
\text { Environment, Health \& } \\
\text { Safety, Education, Grants, } \\
\text { Charitable Contributions }\end{array}$ \\
\hline Cardinal Health Inc. & Health Care & Core values & Community Relations \\
\hline Caterpillar Inc. & $\begin{array}{l}\text { Construction } \\
\text { \& Mining Equipment }\end{array}$ & $\begin{array}{l}\text { Corporate Responsibility } \\
\text { (Social, Environment, } \\
\text { Economic) }\end{array}$ & Sustainable Development \\
\hline
\end{tabular}


L'implicite dans le discours des grandes entreprises sur leur responsabilité sociale

Chevron Corp. Oil \& Gas Social Responsibility

Chubb Corp.

Finance, Insurance

Living the Diversity Vision

Corporate \&

Social Responsibility

Solutions

Finance

Beverages

Technology

Dell Inc.

Dow Chemical Co. Basic Materials

Duke Energy Corp Electric Power

eBay Inc.

Online Marketplace

Exelon Corp.

Utilities

Federated

Department

Stores Inc.

General Electric Co. Power Provider

Goldman Sachs Finance

Group Inc.

Hewlett-Packard Co. Technology
eBay Foundation

Citizenship

Corporate

Citizenship

porate

Citizenship, Our Shared

Responsibilities

Corporate Responsibility

Commitment

Social Responsibility

Community

Social Responsibility

Stakeholder Engagement

Global Leadership and Diversity,

Environmental Policy, Foundation, Charitable Services

Business Ethics, Community engagement, Philanthropy, Supply Chain Responsibility 


\begin{tabular}{|c|c|c|c|}
\hline Intel Corp. & $\begin{array}{l}\text { Information } \\
\text { Technology }\end{array}$ & $\begin{array}{l}\text { Corporate } \\
\text { Responsibility }\end{array}$ & $\begin{array}{l}\text { Workplace of Choice, } \\
\text { Environmental Health \& } \\
\text { Safety, Intel in your } \\
\text { Community, Education } \\
\text { Initiative, Quality, } \\
\text { Diversity, Advancing Lives } \\
\text { across the World }\end{array}$ \\
\hline $\begin{array}{l}\text { International } \\
\text { Business } \\
\text { MachinesCorp. }\end{array}$ & $\begin{array}{l}\text { Information } \\
\text { Technology }\end{array}$ & $\begin{array}{l}\text { Corporate } \\
\text { Responsibility }\end{array}$ & $\begin{array}{l}\text { Citizenship across IBM, } \\
\text { Environment, } \\
\text { Community Relations, } \\
\text { Supply Chain Social } \\
\text { Responsibility, } \\
\text { Employee Well-Being, } \\
\text { Compliance \& Governance }\end{array}$ \\
\hline Johnson \& Johnson & Health Care & $\begin{array}{l}\text { Corporate } \\
\text { Responsibility }\end{array}$ & $\begin{array}{l}\text { Contributions, Diversity, Environment, } \\
\text { Governance, HIV/AIDS, Health \& } \\
\text { Safety, Policies }\end{array}$ \\
\hline $\begin{array}{l}\text { Kimberly-Clark } \\
\text { Corp. }\end{array}$ & Health \& Hygiene & Sustainability & \\
\hline Lowe's Cos. & Home Improvement & Community & $\begin{array}{l}\text { Commitment to Community, Social } \\
\text { Responsibility }\end{array}$ \\
\hline 3M Co. & Global Technology & Sustainability & $\begin{array}{l}\text { Environmental, Social and Economic } \\
\text { Sustainability }\end{array}$ \\
\hline McDonald's Corp. & Foodservice & $\begin{array}{l}\text { Corporate } \\
\text { Responsibility }\end{array}$ & CR Blog \\
\hline Medtronic Inc. & Medical Technology & Philanthropy & $\begin{array}{l}\text { Medtronic in the Community, Health, } \\
\text { Education }\end{array}$ \\
\hline $\begin{array}{l}\text { Merrill Lynch \& } \\
\text { Co. Inc. }\end{array}$ & Finance & Corporate Citizenship & $\begin{array}{l}\text { Corporate Social Responsibility, } \\
\text { Community Development, } \\
\text { Environmental Sustainability }\end{array}$ \\
\hline Microsoft Corp. & $\begin{array}{l}\text { Information } \\
\text { Technology }\end{array}$ & Corporate Citizenship & $\begin{array}{l}\text { Business Conduct, Legal Compliance, } \\
\text { SCM, Responsible Industry, } \\
\text { Environmental Impact /donations, } \\
\text { responsible employer/customers } \\
\text { and partners. }\end{array}$ \\
\hline Motorola Inc. & $\begin{array}{l}\text { Wireless \& } \\
\text { Broadband } \\
\text { Technology }\end{array}$ & Corporate Citizenship & $\begin{array}{l}\text { Business Principles, Governance, } \\
\text { Environment, Health \& Safety, } \\
\text { Human Rights, Policy, Social } \\
\text { Commitment }\end{array}$ \\
\hline PepsiCo Inc. & Foods \& Beverages & Citizenship & $\begin{array}{l}\text { CSR, Sustainability Vision, } \\
\text { Environment, Community, Health \& } \\
\text { Wellness, HIV/AIDS Initiatives }\end{array}$ \\
\hline Pfizer Inc. & Health Care & Corporate Citizenship & Policies \& Practices \\
\hline $\begin{array}{l}\text { Procter \& Gamble } \\
\text { Co. }\end{array}$ & Consumer Goods & Our Commitment & $\begin{array}{l}\text { Social Responsibility, Communities, } \\
\text { Environment, Sustainability }\end{array}$ \\
\hline Schlumberger Ltd. & Oil \& Gas & Glopal Citizensmp & \\
\hline
\end{tabular}


L'implicite dans le discours des grandes entreprises sur leur responsabilité sociale

\begin{tabular}{|c|c|c|c|}
\hline Starbucks Corp. & Consumer Services & $\begin{array}{l}\text { Corporate Social } \\
\text { Responsibility }\end{array}$ & $\begin{array}{l}\text { Communities, Environmental Affairs, } \\
\text { Supplier Diversity, Donations }\end{array}$ \\
\hline Target Corp. & $\begin{array}{l}\text { Consumer } \\
\text { Services }\end{array}$ & $\begin{array}{l}\text { Philosophy } \\
\text { and Values }\end{array}$ & Corporate Governance \\
\hline $\begin{array}{l}\text { Texas Instruments } \\
\text { Inc. }\end{array}$ & Technology & $\begin{array}{l}\text { Corporate Social } \\
\text { Responsibility }\end{array}$ & $\begin{array}{l}\text { Governance, Environment \& Safety, } \\
\text { Health, Diversity, Community, } \\
\text { Education, Ethics, Employee } \\
\text { Well-Being }\end{array}$ \\
\hline Time Warner Inc. & $\begin{array}{l}\text { Global Media \& } \\
\text { Entertainment }\end{array}$ & Citizenship & $\begin{array}{l}\text { Customers, Employees \& Workplace, } \\
\text { Environment, Supply Chain, } \\
\text { Community, Diversity }\end{array}$ \\
\hline $\begin{array}{l}\text { UnitedHealth } \\
\text { Group Inc. }\end{array}$ & Health Care & $\begin{array}{l}\text { Community } \\
\text { Commitment }\end{array}$ & Corporate Governance \\
\hline $\begin{array}{l}\text { United Parcel } \\
\text { Service Inc. }\end{array}$ & $\begin{array}{l}\text { Delivery, } \\
\text { Special } \\
\text { Transportation } \\
\text { \& Logistics }\end{array}$ & Sustainability & $\begin{array}{l}\text { UPS \& the Community, Philanthropy, } \\
\text { Safety, Environment, Diversity, } \\
\text { Education }\end{array}$ \\
\hline $\begin{array}{l}\text { United Technologies } \\
\text { Corp. }\end{array}$ & $\begin{array}{l}\text { Global } \\
\text { Technology }\end{array}$ & $\begin{array}{l}\text { Corporate } \\
\text { Responsibility }\end{array}$ & Commitment, Social Responsibility \\
\hline Walgreen Co. & Drugstores & Community & \\
\hline Walt Disney Co. & $\begin{array}{l}\text { Consumer } \\
\text { Services }\end{array}$ & $\begin{array}{l}\text { Corporate } \\
\text { Responsibility }\end{array}$ & $\begin{array}{l}\text { Business Standards \& Ethics, } \\
\text { Corporate, Governance, } \\
\text { Community, Environmentality, } \\
\text { Safety, International Labour Standards }\end{array}$ \\
\hline
\end{tabular}

ANNEXE 2 - TABLEAU DES 50 ENTREPRISES COMPOSANT LE FTSE4GOOD (UK), FÉVRIER 2007

\begin{tabular}{|c|c|c|c|}
\hline Entreprises & Secteurs & Rubrique principale & Sous-catégories \\
\hline $\begin{array}{l}\text { Alliance } \\
\text { \& Leicester }\end{array}$ & Banking & $\begin{array}{l}\text { CSR and } \\
\text { Governance }\end{array}$ & $\begin{array}{l}\text { Committees, Community Relations, } \\
\text { Shareholder Relations }\end{array}$ \\
\hline $\begin{array}{l}\text { Alliance } \\
\text { Boots }\end{array}$ & $\begin{array}{l}\text { Health \& Beauty } \\
\text { Retailer }\end{array}$ & CSR Website & $\begin{array}{l}\text { Community Environment Marketplace, } \\
\text { Workplace }\end{array}$ \\
\hline AstraZeneca & Pharmaceuticals & Responsibility & $\begin{array}{l}\text { Corporate Responsibility } \\
\text { People, climate change }\end{array}$ \\
\hline Aviva & Insurance & $\begin{array}{l}\text { Corporate Social } \\
\text { Responsibility }\end{array}$ & $\begin{array}{l}\text { Standard of Business Conduct, } \\
\text { Customers, Human } \\
\text { Rights, Workforce, Health \& Safety, } \\
\text { Suppliers, } \\
\text { Communities, Environment }\end{array}$ \\
\hline $\begin{array}{l}\text { BG } \\
\text { Group }\end{array}$ & Gas & $\begin{array}{l}\text { Corporate } \\
\text { Responsibility }\end{array}$ & $\begin{array}{l}\text { Conduct, Our People, Responsible to } \\
\text { Society, Environment }\end{array}$ \\
\hline $\begin{array}{l}\text { BHP } \\
\text { Billiton }\end{array}$ & $\begin{array}{l}\text { Diversified } \\
\text { resources }\end{array}$ & $\begin{array}{l}\text { Sustainability } \\
\text { at BHP Billiton }\end{array}$ & $\begin{array}{l}\text { Health, Safety, Environment, Engaging } \\
\text { Stakeholders, Stewardship }\end{array}$ \\
\hline
\end{tabular}




\begin{tabular}{|c|c|c|c|}
\hline BP & Global Energy & $\begin{array}{l}\text { Environment } \\
\& \text { Society }\end{array}$ & $\begin{array}{l}\text { BP \& Sustainability, BP \& Responsibility, Diversity } \\
\text { \& Inclusion }\end{array}$ \\
\hline $\begin{array}{l}\text { BT } \\
\text { Group }\end{array}$ & Communications & Company Profile & CSR, Our Business Practice \& Code of Ethics \\
\hline Barclays & Banking & $\begin{array}{l}\text { Corporate } \\
\text { Responsibility }\end{array}$ & $\begin{array}{l}\text { Community Investment, Environment, Equality \& } \\
\text { Diversity, Policies \& principles }\end{array}$ \\
\hline $\begin{array}{l}\text { British } \\
\text { Land Co. }\end{array}$ & Property Investment & $\begin{array}{l}\text { Management, } \\
\text { Governance } \\
\text { \& CSR }\end{array}$ & $\begin{array}{l}\text { Environment \& CSR, Workplace, Marketplace, } \\
\text { Community, Environment }\end{array}$ \\
\hline $\begin{array}{l}\text { British } \\
\text { Sky } \\
\text { Brodcasting } \\
\text { Group }\end{array}$ & Media & $\begin{array}{l}\text { Corporate } \\
\text { Responsability }\end{array}$ & Managing our Responsibility Commitments \\
\hline $\begin{array}{l}\text { Cadbury } \\
\text { Schweppes }\end{array}$ & $\begin{array}{l}\text { Beverages and } \\
\text { Confectionary }\end{array}$ & $\begin{array}{l}\text { Society \& } \\
\text { Environment }\end{array}$ & $\begin{array}{l}\text { Our Corporate Social Responsibility in Action, } \\
\text { Our CSR Vision \& Strategy, Goals \& } \\
\text { Commitments on Sustainability }\end{array}$ \\
\hline Carnival & $\begin{array}{l}\text { Global Cruise } \\
\text { Operations }\end{array}$ & $\begin{array}{l}\text { Corporate } \\
\text { governance }\end{array}$ & $\begin{array}{l}\text { Corporate Responsibility } \\
\text { Code of Business Conduct \& Ethics, } \\
\text { Environmental policy; Community Relations }\end{array}$ \\
\hline Centrica & Energy & $\begin{array}{l}\text { Corporate } \\
\text { Responsibility }\end{array}$ & $\begin{array}{l}\text { Climate change, Vulnerable Customers, Health, } \\
\text { Safety \& Security }\end{array}$ \\
\hline $\begin{array}{l}\text { Compass } \\
\text { Group }\end{array}$ & $\begin{array}{l}\text { Global } \\
\text { Foodservice }\end{array}$ & $\begin{array}{l}\text { Responsibility } \\
\text { \& Society }\end{array}$ & $\begin{array}{l}\text { Acting Responsibly, Our Code of Ethics, } \\
\text { Responsibility and Society, Compass in the } \\
\text { Community }\end{array}$ \\
\hline Diageo & $\begin{array}{l}\text { Spirits, Wine and } \\
\text { Beer Business }\end{array}$ & $\begin{array}{l}\text { Corporate } \\
\text { Citizenship }\end{array}$ & $\begin{array}{l}\text { Community Environment, Promoting } \\
\text { Responsible Drinking, Responsible Marketing \& } \\
\text { Innovation, Corporate Governance }\end{array}$ \\
\hline $\begin{array}{l}\text { Experian } \\
\text { Group }\end{array}$ & IT Solutions & Responsibilities & $\begin{array}{l}\text { Corporate Governance } \\
\text { Corporate Responsibility }\end{array}$ \\
\hline $\begin{array}{l}\text { Glaxo } \\
\text { Smith } \\
\text { Kline }\end{array}$ & Pharmaceuticals & Responsibility & $\begin{array}{l}\text { CR Statements and Policies, Business Ethics, } \\
\text { Clinical Trials, Ethical Issues, Stakeholder } \\
\text { Engagement, Human Rights }\end{array}$ \\
\hline HBOS & $\begin{array}{l}\text { Mortgage and } \\
\text { Savings }\end{array}$ & Community & Corporate Responsibility \\
\hline $\begin{array}{l}\text { HSBC } \\
\text { Holdings }\end{array}$ & Banking & CSR at $\mathrm{HSBC}$ & $\begin{array}{l}\text { Our Principles \& Values, Our Stakeholders, } \\
\text { Our Sustainable Approach to Banking, } \\
\text { Environment, People, Community }\end{array}$ \\
\hline $\begin{array}{l}\text { Home } \\
\text { Retail } \\
\text { Group }\end{array}$ & $\begin{array}{l}\text { Home and } \\
\text { General } \\
\text { Merchandise } \\
\text { Retail }\end{array}$ & $\begin{array}{l}\text { Corporate } \\
\text { Responsibility }\end{array}$ & $\begin{array}{l}\text { Defining \& Managing CR, Our Approach, } \\
\text { Home Retail } \\
\text { Group and the Environment / and the Supply } \\
\text { Chain/and the Marketplace }\end{array}$ \\
\hline $\begin{array}{l}\text { Interna- } \\
\text { tional } \\
\text { Power }\end{array}$ & Power & Commitment & $\begin{array}{l}\text { Environment \& Efficiency, People, } \\
\text { Community, Suppliers, CSR Performance }\end{array}$ \\
\hline Kingfisher & $\begin{array}{l}\text { Home } \\
\text { Improvement } \\
\text { Retail }\end{array}$ & $\begin{array}{l}\text { Social } \\
\text { Responsibility }\end{array}$ & $\begin{array}{l}\text { Our Vision, Our Approach, Environmental Issues, } \\
\text { Social } \\
\text { Issues, Charity }\end{array}$ \\
\hline
\end{tabular}


L'implicite dans le discours des grandes entreprises sur leur responsabilité sociale

\begin{tabular}{|c|c|c|c|}
\hline $\begin{array}{l}\text { Land } \\
\text { Securities } \\
\text { Group }\end{array}$ & Property & Responsibility & $\begin{array}{l}\text { Corporate Governance, Environment, Human Resources, } \\
\text { Investor relations, Customer Service, Shareholder Information, } \\
\text { Sponsorships }\end{array}$ \\
\hline $\begin{array}{l}\text { Legal \& } \\
\text { General } \\
\text { Group }\end{array}$ & $\begin{array}{l}\text { Financial } \\
\text { Services }\end{array}$ & CSR & $\begin{array}{l}\text { CSR at Legal \& General, Ethical Values, CSR Governance \& } \\
\text { Management }\end{array}$ \\
\hline $\begin{array}{l}\text { Lloyds } \\
\text { TSB Group }\end{array}$ & Banking & $\begin{array}{l}\text { About Lloyds } \\
\text { TSB }\end{array}$ & $\begin{array}{l}\text { Corporate Responsibility, CR Principles, Marketplace, } \\
\text { Workplace, Community, Environment }\end{array}$ \\
\hline Man Group & $\begin{array}{l}\text { Finance } \\
\text { and Brokerage }\end{array}$ & & Corporate Responsibility Report \\
\hline $\begin{array}{l}\text { Marks \& } \\
\text { Spencer } \\
\text { Group }\end{array}$ & Retail & $\begin{array}{l}\text { Our } \\
\text { Responsibilities }\end{array}$ & $\begin{array}{l}\text { Our Approach to CSR, Customers, Workplace, Investors, } \\
\text { Suppliers, Community Funding, Environment }\end{array}$ \\
\hline $\begin{array}{l}\text { National } \\
\text { Grid }\end{array}$ & $\begin{array}{l}\text { Network } \\
\text { Utilities }\end{array}$ & Responsibility & $\begin{array}{l}\text { Operating Responsibly, Our Impacts, Managing Our Impacts, } \\
\text { Our Stakeholders }\end{array}$ \\
\hline Old Mutual & $\begin{array}{l}\text { Financial } \\
\text { Services }\end{array}$ & $\begin{array}{l}\text { Corporate } \\
\text { Responsibility }\end{array}$ & $\begin{array}{l}\text { Black Economic Empowerment, Code of Conduct Ethics, } \\
\text { Environmental Policy, Health \& Safety, } \\
\text { Social Investment }\end{array}$ \\
\hline Pearson & Media & Community & $\begin{array}{l}\text { Our Business \& Society, Our Communities, Our people, } \\
\text { Diversity; Labour Standards \& Human Rights, Environment, } \\
\text { Managing Our Risks }\end{array}$ \\
\hline Prudential & $\begin{array}{l}\text { Financial } \\
\text { Services }\end{array}$ & $\begin{array}{l}\text { Corporate } \\
\text { Responsibility }\end{array}$ & $\begin{array}{l}\text { Global issues, Community Programmes, Management } \\
\text { Policies, Sustainable Development }\end{array}$ \\
\hline $\begin{array}{l}\text { Reckitt } \\
\text { Benckiser }\end{array}$ & $\begin{array}{l}\text { Household } \\
\text { Cleaning, \& } \\
\text { Personal Care }\end{array}$ & $\begin{array}{l}\text { Corporate } \\
\text { Responsibility }\end{array}$ & $\begin{array}{l}\text { Vision \& Values, Code of Business Conduct, Environment, } \\
\text { Social, Health \& Safety, Community involvement, } \\
\text { Global manufacturing Standard, Animal testing }\end{array}$ \\
\hline $\begin{array}{l}\text { Reed } \\
\text { Elsevier }\end{array}$ & Media & $\begin{array}{l}\text { Corporate } \\
\text { Responsibility }\end{array}$ & $\begin{array}{l}\text { Governance, Marketplace, Workplace, } \\
\text { Community, Environment }\end{array}$ \\
\hline $\begin{array}{l}\text { Reuters } \\
\text { Group }\end{array}$ & $\begin{array}{l}\text { Global } \\
\text { Information }\end{array}$ & $\begin{array}{l}\text { CSR \& } \\
\text { Governance }\end{array}$ & $\begin{array}{l}\text { Corporate Responsibility, Community, } \\
\text { Our Approach, Environment, Marketplace, Workplace }\end{array}$ \\
\hline $\begin{array}{l}\text { Royal } \\
\text { Bank of } \\
\text { Scotland } \\
\text { Group }\end{array}$ & Banking & $\begin{array}{l}\text { Corporate } \\
\text { Responsibility }\end{array}$ & $\begin{array}{l}\text { Managing Our Business Responsibility, Policies \& External } \\
\text { Commitments, Community Investment, Environment }\end{array}$ \\
\hline $\begin{array}{l}\text { Royal Dutch } \\
\text { Shell A/B }\end{array}$ & $\begin{array}{l}\text { Oil, Energy and } \\
\text { Petrochemicals }\end{array}$ & $\begin{array}{l}\text { Environment \& } \\
\text { Society }\end{array}$ & $\begin{array}{l}\text { Sustainable Development \& Business Strategy, Global } \\
\text { Environmental Issues, Living by our Principles, The } \\
\text { World We Live In. }\end{array}$ \\
\hline SABMiller & Brewery & $\begin{array}{l}\text { Our } \\
\text { Responsibility }\end{array}$ & $\begin{array}{l}\text { Our Commitment, Responsible Drinking, } \\
\text { Our Approach to Sustainable Development, } \\
\text { Customer satisfaction, Black Economic Empowerment }\end{array}$ \\
\hline Sainsbury & Food Retail & $\begin{array}{l}\text { Corporate } \\
\text { Responsibility }\end{array}$ & Our Approach, Principles \\
\hline $\begin{array}{l}\text { Scottish } \\
\text { \& Newcastle }\end{array}$ & Brewery & Responsibility & $\begin{array}{l}\text { Corporate Responsibility in Scottish \& } \\
\text { Newcastle, Corporate Responsibility in Context, } \\
\text { Responsible Drinking, Our People, Community, } \\
\text { Environment }\end{array}$ \\
\hline
\end{tabular}




\begin{tabular}{|c|c|c|c|}
\hline $\begin{array}{l}\text { Scottish \& } \\
\text { Southern } \\
\text { Energy }\end{array}$ & $\begin{array}{l}\text { Energy, } \\
\text { Electricity }\end{array}$ & $\begin{array}{l}\text { Corporate } \\
\text { Responsibility }\end{array}$ & $\begin{array}{l}\text { Our Values, Our Performance, Corporate Governance, } \\
\text { Energy Efficiency, Education, Community }\end{array}$ \\
\hline $\begin{array}{l}\text { Scottish } \\
\text { Power }\end{array}$ & Energy & $\begin{array}{l}\text { Corporate } \\
\text { Responsibility }\end{array}$ & $\begin{array}{l}\text { Managing Our Responsibilities, Governance, } \\
\text { Stakeholder Engagement, Our } 12 \text { Impacts, } \\
\text { Environment, Renewables }\end{array}$ \\
\hline $\begin{array}{l}\text { Smith \& } \\
\text { Nephew }\end{array}$ & $\begin{array}{l}\text { Global Medical } \\
\text { Technology }\end{array}$ & $\begin{array}{l}\text { Sustainability } \\
\text { Report }\end{array}$ & Social Responsibility, Managing Principles \\
\hline $\begin{array}{l}\text { Standard } \\
\text { Chartered }\end{array}$ & Banking & $\begin{array}{l}\text { Corporate } \\
\text { Responsibility }\end{array}$ & $\begin{array}{l}\text { Our Approach, People, Environment, } \\
\text { Communities, Customers, Suppliers }\end{array}$ \\
\hline Tesco & Grocery & $\begin{array}{l}\text { Corporate } \\
\text { Responsibility }\end{array}$ & $\begin{array}{l}\text { Our Approach, Economy, Society, Charities \& } \\
\text { Fundraising, Environment }\end{array}$ \\
\hline Unilever & $\begin{array}{l}\text { Home, Personal } \\
\text { Care \& Food } \\
\text { Products }\end{array}$ & $\begin{array}{l}\text { Our Values, } \\
\text { Environment \& } \\
\text { Society }\end{array}$ & $\begin{array}{l}\text { Managing Corporate Responsibility, Living Out our } \\
\text { Values, Governance, Engaging with Stakeholders, } \\
\text { Reporting }\end{array}$ \\
\hline $\begin{array}{l}\text { United } \\
\text { Utilities }\end{array}$ & Utilities & $\begin{array}{l}\text { Corporate } \\
\text { Responsibility }\end{array}$ & $\begin{array}{l}\text { How We Work, Business Benefits, Sustainability, } \\
\text { Supply Chain }\end{array}$ \\
\hline $\begin{array}{l}\text { Vodafone } \\
\text { Group }\end{array}$ & Mobile Telecom & $\begin{array}{l}\text { Corporate } \\
\text { Responsibility }\end{array}$ & $\begin{array}{l}\text { Managing CR, Stakeholder Engagement, Socio- } \\
\text { economic Impact, Earning the Trust of Customers, } \\
\text { Environment, Supply Chain, Employees, Foundations } \\
\text { Commitments }\end{array}$ \\
\hline $\begin{array}{l}\text { WPP } \\
\text { Group }\end{array}$ & $\begin{array}{l}\text { Communications } \\
\text { Services }\end{array}$ & $\begin{array}{l}\text { About Us, How } \\
\text { We Behave }\end{array}$ & $\begin{array}{l}\text { Corporate Responsibility, CR Policy, Employment, } \\
\text { Marketing Ethics, Social Investment, Supply Chain, } \\
\text { The Impact of our Work }\end{array}$ \\
\hline Wolseley & $\begin{array}{l}\text { Construction } \\
\text { Products }\end{array}$ & $\begin{array}{l}\text { Corporate } \\
\text { Responsibility }\end{array}$ & Corporate Responsibility Report \\
\hline
\end{tabular}

\section{ANNEXE 3 -RAPPORTS DES ENTREPRISES SUR LEURS RESPONSABILITÉS}

TYPES DE RAPPORT

Business and Society Report

Citizenship Report

Community Resources Report

Corporate Citizenship Report

Corporate (and) Social

Responsibility Report

Corporate Responsibility Report

\section{ENTREPRISES UK ET US}

Pearson

General Electric

Prudential

Old Mutual, Diageo Motorola

Alliance \& Leicester, Alliance Boots, Aviva, Cadbury

Schweppes, HSBC, Legal \& General Group, Marks \& Spencer, National Grid, Pearson, Wolseley

Allstate, Chubb, Intel, Pfizer, Starbucks, Time Warner

Astrazeneca, Barclays, BG Group, British Land, BSkyB, Centrica, Experian, GlaxoSmithKlyne, HBOS, Home Retail Group, Land Securities, Lloyds, Man Group, Prudential, Reed Elsevier, Royal Bank of Scotland, Sainsbury, Scottish \& Newcastle, Scottish \& Southern Energy, Standard Chartered, Tesco, United Utilities, Vodafone, WPP

Aetna, Adobe, Cisco, Citigroup, IBM, Intel, McDonald's,

Target, United Technologies 
L'implicite dans le discours des grandes entreprises sur leur responsabilité sociale

\author{
Corporate Sustainability Report \\ Environmental and Social Report \\ Environment, Safety \& Health \\ Operations Report
}

\section{Global Citizenship Report}

Social and Environmental Report

Social Responsibility Report

Sustainability Report

Sustainability and Philanthropy Report

Sustainable Development Report
UPS

Unilever

Texas Instruments

Old Mutual

Abbott, Hewlett Packard, Microsoft, Schlumberger

$B T$

Kingfisher

Lowe's

BHP Billiton, BP, Reckitt Benckiser, Royal Dutch Shell, Smith \&Nephew

Alcoa, Baxter, Dell, Johnson \& Johnson, Kimberley-Clarke, $3 \mathrm{M}$, PepsiCo

Procter \& Gamble

SABMiller

ANNEXE 4 - Titres SPÉCIAUX POUR LES RAPPORTS 2005 OU 2006

FTSE4GOOD UK 50

Alliance Boots: Why Boots Believes a Responsible Business is a Healthy Business (Corporate Responsibility Report)

Aviva : Moving Forward in CSR (Corporate Responsibility Report)

Barclays: Responsible Banking (Corporate Responsibility Report)

BG Group : Corporate Responsibility is at the Heart of our Business (Corporate Responsibility Report)

BHP Billiton: Licence to Operate (Sustainability Report)

BP: Making Energy More (Sustainability Report)

BT: Changing World: Sustained Values (Social and Environmental Report)
DFSI US 50

Abbott: Changing Lives
(Global Citizenship Report)

Aetna: Demonstrating Social Responsibility and Integrity (Corporate Social Responsibility Report)

Allstate: How does CSR

- Protect and Prepare Customers?

- Bring Out the Best in Employees?

- Help Communities Thrive?

- Enhance our Company's Value?

(Corporate Social Responsibility Report)

Baxter: 75 Years of Saving and Sustaining Lives (Sustainability report)

Cisco : Cisco and Corporate Citizenship (Corporate Responsibility Report)

Citigroup: Citizenship

(Corporate responsibility Report)

Dow Chemicals : This is Dow...

One Part Vision

One Part Foundation

One Part Aspiration

One Part Inspiration

One Part Action

(Corporate Report) 
Cadbury Schweppes: Living our Values:

Yesterday, Today, Tomorrow

Corporate Social Responsabilities Report

Centrica: Investing in Sustainability

(Corporate Responsibility Report)

Diageo: Celebrating Life, Everyday, Everywhere

(Corporate Citizenship Report)

HSBC: Future History

(Corporate Social Responsibility Report)

Kingfisher: Steps to Responsible Growth

(Social Responsibility Report)

Man Group : Our Reputation...Is Held in Trust by our People

(Corporate Responsibility Report)

Marks \& Spencer: Your M \& S

(Corporate Social Responsibility Report)

Prudential : Acting Responsibly

(Corporate Responsibility Report)

Sowing Seeds, Growing Futures

(Community Resources Report)

Reed Elsevier: Making an Impact

(Corporate Responsibility Report)

Royal Bank of Scotland: Make It Happen

(Corporate Responsibility Report)

Royal Dutch Shell : Meeting the Energy Challenge (Sustainability Report)

Scottish \& Southern Energy : Powerful Opportunities (Corporate Responsibility Report)

Standard Chartered : Making a World of Difference ... to Stakeholders through Sustainable Business Practices (Corporate Responsibility Report)

Vodafone: We Said, We Have, We Will

(Corporate Responsibility Report)

Wolseley: Building Success

(Corporate Social Responsibility Report)
Exelon: Realizing the Promise

(Progress Report: Environnement, Safety, Community)

General Electric: Solving Big Needs (Citizenship Report)

IBM: Collaboration That Matters (Corporate Responsibility Report)

Intel : Let's Be Clear/ Our Commitment to Corporate Responsibility (Corporate Responsibility Report)

Johnson \& Johnson : Engaging More People (Sustainability Report)

Kimberley-Clarke : Our Commitment to Health \& Hygiene

(Sustainability Report)

Lowe's: More than a Store

(Social Responsibility Report)

Medtronic: Everyday, Every Way, Everyone (Code of Conduct)

Motorola: We Create Possibilities

(Corporate Citizenship Report)

PepsiCo: Tomorrow > Today

(Sustainability Report)

Starbucks: My Starbucks

(Corporate Social Responsibility Report)

UPS : Operating in Unison (Corporate Sustainability Report)

ANNEXE 5 -FrÉQUENCES LEXICALES

SUBSTANTIFS LES PLUS FRÉQUEMMENT UTILISÉS PAR ORDRE DÉCROISSANT

FTSE4GOOD UK 50

Business / Company

Customer

People
DFSI US 50

Business / company

Community

Employee 
L'implicite dans le discours des grandes entreprises sur leur responsabilité sociale

Value / values

Community

Employee

Commitment

Stakeholder

Society

Shareholde / World
World

People

Commitment

Customer

Stakeholder

Value / values

Compliance

\section{ANNEXE 6 - LES 10 PRINCIPES DU GLOBAL COMPACT DE L'ONU}

$<$ www.novethic.fr>

« Cette initiative, lancée en 1999 par l'ONU, consiste à fédérer les Nations Unies, les ONG et les milieux d'affaires autour de quelques grands engagements partagés pour que soient pris en compte les impacts sociaux et environnementaux de la mondialisation.»

1. Soutenir et respecter la protection internationale des droits de l'homme de leur sphère d'influence.

2. Etre sûr que leurs propres entreprises ne se rendent pas complices de violations des droits de l'homme.

3. Respecter la liberté d'association et reconnaître le droit à la négociation collective.

4. Eliminer toutes formes de travail forcé ou punitif.

5. Abolir réellement le travail des enfants.

6. Eliminer toute discrimination dans le recrutement ou l'affectation des postes.

7. Adopter une approche de précaution face aux défis environnementaux.

8. Mener des initiatives pour promouvoir une meilleure responsabilité sociale.

9. Encourager le développement et la diffusion de technologies respectueuses de l'environnement.

10. Lutter contre la corruption. 